\title{
USING EXPERT MODELS IN HUMAN RELIABILITY ANALYSIS - A DEPENDENCE ASSESSMENT METHOD BASED ON FUZZY LOGIC
}

\author{
L. Podofillini, V.N. Dang, E. Zio, P. Baraldi, M. Librizzi
}

\section{Abstract}

In Human Reliability Analysis (HRA), dependence analysis refers to assessing the influence of the failure of the operators to perform one task on the failure probabilities of subsequent tasks. A commonly used approach is the Technique for Human Error Rate Prediction (THERP). The assessment of the dependence level in THERP is a highly subjective judgment based on general rules for the influence of five main factors. A frequently used alternative method extends the THERP model with decision trees. Such trees should increase the repeatability of the assessments but they simplify the relationships among the factors and the dependence level. Moreover, the basis for these simplifications and the resulting tree is difficult to trace. The aim of this work is a method for dependence assessment in HRA that captures the rules used by experts to assess dependence levels and incorporates this knowledge into an algorithm and software tool to be used by HRA analysts. A Fuzzy Expert System (FES) underlies the method. The method and the associated expert elicitation process are demonstrated with a working model. The expert rules are elicited systematically and converted into a traceable, explicit, and computable model. Anchor situations are provided as guidance for the HRA analyst's judgment of the input factors. The expert model and the FESbased dependence assessment method make the expert rules accessible to the analyst in a usable and repeatable means, with an explicit and traceable basis.

Keywords: human reliability analysis; human action dependence; expert judgment; fuzzy expert system; expert elicitation.

Acronyms / Abbreviations

DT - decision tree

FL - fuzzy logic

HRA - Human Reliability Analysis

PSA - Probabilistic Safety Assessment

Prediction
FES - fuzzy expert system
HFE - human failu re events
MF - membership function
THERP - Technique for Human Error Rate 


\section{Introduction}

Expert judgment is required in many areas of risk analysis, where the relevant factors and their relationships are complex and the data are insufficient as a basis for either a statistical estimate or for constructing an empirical model with which estimates may be obtained. Two ways of using expert judgment can be distinguished. The first way relates to collecting and aggregating the judgment of experts on the variables of interest, e.g. a failure probability or a seismic hazard curve (e.g. Cooke, 1991). Formal approaches for this exist, which are very useful to bring out the assumptions and reasoning underlying the judgments and to document them so that they can be appraised by others (Cooke, 1991; O'Hagan et al., 2006). However, some disadvantages are the cost and time they require and the potential presence of biases in the expert estimates (for a complete discussion see: Otway \& von Winterfeldt, 1992). A second way of using expert judgment is based on collecting information from experts to build a computable model (called the expert model) with which the desired value may be obtained. Examples include the computerized diagnostic aids used in nuclear power plants, e.g. Chang et al. (1995), and clinical decision support systems used in medicine, e.g. Kaw amoto et al. (2005).

The work reported here addresses an application of expert judgment in HRA, the part of PSAs that deals with human performance and its impact on risk. In HRA, dependence analysis refers to assessing the influence of the failure of the operators to perform one task on the failure probabilities of subsequent tasks (Swain \& Guttman, 1983). In qualitative terms, a dependence is said to exist between two tasks, that is two Human Failure Events (HFEs) if the failure of the second HFE is more likely given that the operators have failed in their performance of the first HFE than following success of the first HFE.

The assessment of dependence has a significant impact on the overall results of a PSA, since the dependent failure probability may be an order of magnitude or more larger than the independent one. An appropriate assessment of dependence is thus essential to avoid underestimation of the risk and to ensure a realistic risk profile from the PSA results. In identifying the HFEs for which dependence should be considered, i.e. the scenarios in which multiple HFEs appear, a common practice is to use large screening probabilities for the HFEs. If the probabilities estimated without accounting for dependence are used, the relevant sequences (with multiple HFEs) may be truncated. A quantification of the scenarios without consideration of dependencies may miss candidates for potential depend encies (NUREG-1792, 2005). 
The development of an expert model for HRA dependence assessment and an assessment method based on this model is aimed at increasing the repeatability of these assessments. The expert model can systematically and transparently represent the assumptions and rules underlying the assessment; at the same time, it can represent relatively complex assessment rules that account for the interactions among the input factors. The attractiveness of a method based on an expert model is that it makes the expert knowled ge and rules accessible to an HRA analyst. Since dependence assessments are needed within each HRA (each PSA study), such a method can circumvent the need to convene an expert or experts in a formal elicitation for each study.

This work focuses on dependencies among post-initiator HFEs. In current PSAs, the dependence model from the Technique for Human Error Rate Prediction (THERP) HRA method (Swain \& Guttman, 1983) is commonly used. It has two parts: a qualitative assessment of a dependence level, ranging in discrete steps from zero (independent tasks or actions) to complete dependence, and the quantification of the impact of the assessed dependence level on the conditional probability of the subsequent task based on a set of formulas. The THERP model refers to five main factors: spatial relatedness, time relationship, functional relatedness, stress, and the similarities among the personnel performing the tasks. While the THERP dependence model provides general guidelines for the influence of these factors on the dependence level (cf. Table 10-1 in Swain \& Guttman, 1983), the assignment of the level is essentially a direct expert judgment, a highly subjective process that can be weak in terms of transparency and repeatibility. The ASME Standard for Probabilistic Risk Assessment notes that "the state of the art in HRA is such that the assessment of dependency is largely based on the analyst's judgement." (Note (1) to HR-G7, ASME, 2005).

To address these issues and reduce the subjectivity inherent in judging the dependence level directly, the assignment of the dependence level is frequently supported with decision trees (DTs), for instance, in the SPAR-H (Gertman et al., 2005) method, in the EPRI HRA “calculator"(Julius et al., 2005), as well as in the recently presented method DEPEND-HRA (Cepin, 2006, 2008a, 2008b). However, the decision tree representation frequently very much simplifies the relationships among the input factors and the dependence level. In addition, the basis for the decision tree is difficult to trace. It should be noted that although the mentioned methods have been specifically developed for nuclear power plants applications, human failure dependence assessment is an important part of the HRA for any technical system (Kennedy et al., 2007). 
Current practice has a number of weaknesses. The absence of specific guid ance makes the use of the THERP dependence method difficult and the results may lack traceability and repeatability. This also makes the review of the assessment by a second person difficult (e.g. in peer or regulatory reviews). The use of DTs improves the situation: the analyst has to give judgments on the input factors, but is not required to draw conclusions on the dependence level, which the DT yields. The central idea is that the input factors should be less subjective quantities than the dependence level (optimally, they should be "measurable"). Yet, DTs are not flexible in the sense that the analyst judgments are typically constrained to rigid options, which refer to extreme situations (Yes/ No, High/ Low). Moving aw ay from binary options also increases the number of branches and the combinations of factors to evaluate. Moreover, different implementations of DTs exist, which may produce significantly different results (Cepin, 2008c): since DTs are often not built from a traceable process of expert elicitation, it is difficult to understand the reasons if two DTs give different results. Section 2 discusses these shortcomings in more detail.

Note that another recent subject of research related to dependence assessment is on how dependent HFEs should be incorporated in large system fault tree analysis (Vaurio, 2000). This subject relates to dependencies among pre-initiator HFEs and a more detailed discussion is outside the scope of the present paper. Also related to dependence assessment is the idea of the existence of human performance limiting values (HPLV) (Kirwan, 2008). It may be the case that accident sequences have very low joint human error probability (e.g. $10^{-4}$ or $10^{-5}$ ), even after dependence is evaluated: the idea is that HPLV should be applied to include for possibly overlooked error mechanisms or error-inducing conditions.

The aim of this work is a method for dependence assessment in HRA that captures the rules used by experts to assess dependence levels and incorporates this knowledge into an algorithm and software tool to be used by HRA analysts. The Fuzzy Expert System (FES) formalism underlies the method. A FES collects the experts' knowled ge as a set of Fuzzy Logic (FL) rules that are mathematically manipulated by Fuzzy Set theory (Zadeh, 1965). Fuzzy set theory has been exploited for HRA in a number of applications (Terano et al., 1983; Onisaw a, 1988a, 1988b; Liang \& Wang, 1993; Kim \& Bishu, 1996; Suresh et al., 1996; Huang et al., 1996; Richei et al., 2001; Konstandinidou et al., 2006; Marseguerra et al., 2006). In most of these, the focus is on using FL to convert human error context descriptions into inputs for existing HRA methods, with the aim of accounting for ambiguity and subjectivity of the descriptions. For example, in Konstandinidou et al. (2006) and Marseguerra et al. (2006), fuzzy logic is applied to 
compute HEPs via the CREAM method, by converting the characterization of the performance shaping factors into fuzzy numbers. Only in Richei et al. (2001) is the problem of building a FES from the expert knowledge also addressed.

At this stage, the focus of the work has been to investigate the suitability and practicality of the FES representation for an HRA dependence assessment method for post-initiator HFEs. This paper presents the basic concepts of the proposed method and demonstrates the approach using a working model of the dependence relationships. The working model is intended to represent a set of moderately complex relationships among the input factors and the dependence level, which could be expected from an expert elicitation. These relationships represent one possible interpretation of the THERP dependence guidelines, but one with more detail. It admittedly does not include all relevant factors but its complexity is sufficient for the purpose of demonstrating the methodology. The details of the FES model are reported in a companion paper (Zio et al., 2009).

To illustrate its use, the FES-based has been applied for dependence assessment of a pair of operator actions in response to an accident scenario in a Boiling Water Reactor. The FES-based method for assessing dependence has the advantage of being able to represent fully the experts' rules (in this case, the rules of the working model), including rules for the interaction of the dependence (input) factors. With anchor situations provided as guidance for the HRA analyst's judgment of the input factors, the method yields the dependence level based on the expert rules. An expert elicitation to obtain a comprehensive set of rules to replace the working model is planned for future work.

The paper is organized as follows. Section 2 gives an overview of the problem of dependence assessment, of the current practice and limitations. Section 3 presents the features of the proposed dependence assessment method. The approach for building the underlying FES-based model is presented in Section 4. Section 5 presents an application of the method. Section 6 discusses traceability, repeatability, verification and valid ity issues.

\section{Dependence assessment in HRA: practice and limitations}

\subsection{The dependence assessment method in THERP}

The dependence assessment method in the THERP HRA method (Swain \& Guttman, 1983 ) is one of the most widely used in the PSA practice. Referred to as the "THERP 
method" in this paper, this dependence assessment method has the following main components:

- Use of conditional human error probabilities (HEPs) to model the effect of dependence: the THERP approach amounts to evaluate the probability of failure of one task, when it is known that the previous task has failed.

- Discretization of the conditional HEP into five ranges representing different levels of dependence: zero, low, moderate, high, complete.

- A formula for computing the dependent, conditional probability for each dependence level. For a low level of dependence, the formula produces for low values of the independent HEP (i.e. $<0.01$ ) a nominal conditional probability value of 0.05 with lower and upper bounds of 0.015 and 0.15 , respectively.

- Guidelines for assessing the level of dependence (summarized in Table 10-1 of Swain \& Guttman (1983)).

The user of the method must analyze the pair of successive tasks and assess the level of dependence. To support the analysis of the tasks dependence, the THERP guidelines suggest the factors that should be considered (THERP Table 10-1 of Swain \& Guttman (1983)): closeness in time and space, functional relatedness (e.g. tasks related to the same subsystem), stress, similarity of the performers (status, training, responsibility, and „many social and psychological factors“).

For example, for the factor closeness in time and space, the guideline reads (item 3 of Table 10-1 of Swain \& Guttman (1983)):

"Evaluate the spatial and time relationship among all events. Dependence between any two events increases as the events occur closer in space and time. For example, displays or controls that are physically close to each other or that must be manipulated at about the same time have a higher level of dependence than items that are widely separated either spatially or as to the time of their manipulation."

These guidelines cannot be used systematically and consistently as a basis for assessing the dependence level because a lot of room is left to interpretation. This makes the assessment a rather difficult task, requiring a considerable amount of expert judgment, which may lack transparency and traceability and leads to low repeatability of the results. Another problem with the direct elicitation of probability is the presence of biases, of many types (Cooke, 1991; Otway, H. \& von Winterfeldt, D., 1992). 


\title{
2.2 Supporting the THERP model with decision trees
}

The expert judgment assessment of the level of dependence is in practice often supported with a decision tree (DT). In these cases, the quantitative impact of the assessed dependence level is still modeled with the THERP dependence assessment method.

Repeatability should improve when expert judgment is structured and supported by a DT. The analyst has to give judgments on the input factors, but is not required to draw conclusions on the dependence level, which comes from the model. An example is shown in Figure 1, which reports the SPAR-H DT for post-initiator HFEs (Gertman et al., 2005). The in put factors of the model are (Figure 1):

- Crews (Same/ Different)

- Time (Close in time/ Not close in time)

- Location (Same/ Different)

- Cues (Additional cues/ No additional cues).

Different implementations of DTs exist. Cepin (2008b) shows that this can lead to significant differences in the result of the HRA and in the evaluation of the risk contributors. Since a traceable process of expert elicitation is often missing, it is difficult to understand the reasons if two DTs give different results.

Another limitation is that DTs are not flexible and may not allow assessments to be representative of the context under analysis. The need to avoid an excessive branching of the tree usually limits the number of branches per factor to two to three values (labels). These labels represent extreme Yes/ No conditions (see Figure 1 for example), which may be difficult to match to a particular context. Indeed, there may be consensus on considering a separation by 5 minutes as "close in time" and one of hours as "not close in time". However, a separation of 20 minutes may be difficult to match to any of the two options, thus requiring a more intermediate judgment that would better represent the context.

\section{INSERT Figure 1. The SPAR-H dependence decision tree}

\author{
INSERT Table 1. The EPRI HRA Calculator ® dependence \\ decision tree (Grobbelaar et al., 2005).
}




\section{Basic concepts of the dependence assessment method}

Figure 2 shows a high-level overview of the dependence assessment method. The underlying FES model and the expert elicitation process for its construction are presented in the next Section 4. The mathematical details of the FES can be found in a companion paper (Zio et al., 2009). The different components of the method are described next.

\section{INSERT Figure 2. Overview of the dependence model. Different models are needed for pre-initiators, and different types of post- initiators.}

\subsection{Input factors}

Similarly to the DTs approach, each of the input factors is represented by a linguistic variable, qualified in terms of linguistic labels (Figure 3). For example: input factor "similarity of performers" may be qualified in terms of a linguistic variable with linguistic labels: None - Low - Medium ... - Very high. Unlike with DTs, the number of linguistic labels for each input factor is higher than two, thus giving more flexibility to the input judgments.

At the same time, the use of a higher number of linguistic labels may become a source of variability in the inputs, so that concrete guidance is needed for the analyst judgments. As shown in Figure 3, this is provided through anchor points that represent prototype conditions of the input factors for a typology of tasks. Different dependence models and thus different sets of anchors may be used for pre-initiators, different types of post initiators, etc. The selection of the proper anchors and their characterization in terms of the linguistic labels is one of the outputs of the expert elicitation process.

Note that numerous studies on expert judgment elicitation have show $n$ that the use of anchors may be counterproductive and bias the judgments if the underling scale is continuous (see Brewer \& Chapman, 2002 for an example, among many others, of a paper discussing the anchor effect, and Stevens, 1946, for the definitions of the measurement scales). However, in our case the anchors are essential because the scale on which they are placed is very abstract for the analyst (what does high "similarity of cues" mean? or what does a similarity of performers of 0.33 mean?). Anchors therefore are necessary to provide reference situations that can orient an analyst. 
With respect to the context characterization, the analyst can provide judgments on the input factors in different ways, for example on a scale (Figure 3). Four anchors are shown in the figure: the analyst may provide a point input on the scale (input 1), or the range where his/ her belief belongs (input 2).

A feature of FES is that they allow overlapping of the linguistic labels (overlapping horizontal bars in Figure 3) to represent the fact that in the common perception, the transition between the linguistic concepts associated to the labels (e.g. between "medium" and "high") is not crisp, but often uncertain and ambiguous. This can be formally accounted for by introducing overlapping fuzzy sets to represent the input variables. Figure 4 shows a possible association of fuzzy sets and trapezoidal membership functions. Note that the association is not shown to the analyst who interfaces only with anchor points and linguistic labels.

Indeed, the natural scale for the input factors is continuous and the discretization introduced by the overlapping labels is a simplification. Yet, this does not seem to be a limitation. The five-level scale is actually attractive because experts and analysts are already very familiar with it from the five THERP levels. Furthermore, as it will be presented in the next Section 3.2, the basis of the fuzzy logic model is a set of rules. This is a concept with which experts and analysts should be already familiar with, since rules (although much coarser) are at the basis of DTs as well.

\section{Insert Figure 3. Analyst elicitation on anchored scale; two analyst input types are shown: as a point value (arrow) and as an interval (horizontal bar).}

\section{Insert Figure 4. Possible association of fuzzy sets and membership functions (trapezoids) to the input qualitative variable.}

\subsection{The underlying model}

The inference model represents the relations between the input factors and the dependence level. This represents the expert knowledge, which in FES is modeled into a set of rules capturing the relationships between the different values of the input and output variables. An example rule reads as follows:

IF

"Factor 1" is "Low" AND "Factor 2" is "Medium" AND ... "Factor N" is 
"High"

THEN

Dependence is "High"

The next Section presents the expert elicitation process to convert the expert knowledge into fuzzy rules.

The input judgments of the analysts are converted into fuzzy numbers, which represent the degree to which the judgments match each of the qualitative labels. The fact that a judgment can match, with different degrees, multiple labels allows FES to handle uncertain and ambiguous statements. Multiple rules are then activated, with a degree that follows from the degree to which each input statement matches the labels involved in the rule. The degree of activation of the rules is then the basis for the derivation of the output of the model, which, as described in the next Section 3.3, is represented by degrees of belief in the different dependence levels. The FL procedure used in this work to associate the output of the model to a given input assessment is based on the Mamdami fuzzy logic (Zio et al., 2009). Accordingly, the degree to which an input assessment matches a label involved in a rule is computed as the maximum value of the intersection of the input assessment and label fuzzy sets (Figure 5, left), the degree of activation of a rule is computed as the minimum value of the degree to which each input assessment matches the labels involved in the rules (Figure 5, right) and the contribution to the output of a given rule is the minimum value between the rule degree of activation and the fuzzy set in the rule output (Figure 5, right). Finally the output fuzzy set is obtained by taking the union of all the involved rule outputs.

Insert Figure 5. Left: example of computation of the degree to which the Factor 1 input assessment matches the label "Low" and the Factor 2 input assessment the label "High" . Right: computation of the degree of activation of the rule If "Factor 1 " is "Low" and "Factor 2" is "High" THEN "Dependence" is "Medium" as minimum value between the degrees to which Factor 1 and Factor 2 input assessments match the corresponding labels in the rule.

\subsection{Outputs}

From the input factors describing the context of two successive actions with respect to their failure dependence, the method produces two outputs. The first consists in the degrees of belief (expressed in terms of possibility) for the different dependence levels. The THERP dependence levels are used (None, Low, Medium, High, Complete). This output shows how the analysts' judgments translate into a possibilistic distribution of the dependence levels. 
The second type of output gives quantitative figures to the dependence level. This output is the expected value of the conditional probability and its associated uncertainty. These are the figures that are included in to the PSA.

The operation that allows passing from the first type of output to the second is called defuzzification (Zadeh, 1965) and, to be formalized, entails associating fuzzy sets and degrees of membership to the levels of dependence.

In the method proposed in this paper, this is done by eliciting information from the THERP handbook. In particular, Table 20-21 of (Swain \& Guttman, 1983) suggests nominal values and uncertainty bounds for the conditional probability associated to each dependence level. A possible association of fuzzy sets to the THERP dependence levels consists in taking membership functions with triangular shape in log 10 scale centered on the associated dependence level nominal values and with supports given by the lower and upper confidence bounds, as reported in Figure 6.

Note that the presented method is still based on the five THERP dependence levels, although a finer characterization of the dependence level could have been obtained with more levels or by adopting a continuous dependence scale (for example, using Bayesian belief nets). However, it was decided to use the THERP dependence levels and the THERP relationships between the levels and the conditional HEPs because these are familiar to experts and analysts. This is an important point for the acceptance of the method.

\author{
Insert Figure 6. Fuzzy sets and membership functions associated \\ to the THERP dependence levels. Elicited from THERP guidelines \\ (Swain \& Guttman, 1983).
}

\title{
4 Building the dependence model: example on a working model
}

The concepts of the elicitation process are here illustrated on a working model of dependence, applicable for post-initiator HFEs of a nuclear power plant for full-power scenarios. The actual expert elicitation exercise will be performed in subsequent work.

The information that need s to be provided by the expert is:

- The important factor relevant to dependence and how they relate

- Selection and positioning of the anchors 
- Impact of the factors on the dependence level

\subsection{Determining input factors and anchors}

The first information to elicit from the expert concerns the important factors for dependence and how they relate. An influence diagram like the one in Figure 7 is the result: it shows the working model used to explore the methodology.

Table 2 lists the input factors, the qualitative labels and the anchor points used in the working model. The expert has to locate the anchors on the input scale, so as to build the input interface of Figure 3.

According to the working model, three factors directly impact the dependence level (Figure 7): "closeness in time", "task relatedness" and "similarity of performers". "Tasks relatedness" is further specified in terms of the "similarity of cues" and "similarity of functions/ goals", as Figure 7 shows. These factors include most, but not all, of the relevant factors. For example, the use of the same procedures may also have influence on the dependence between two tasks. On the other hand, this working model was considered to be of enough complexity for this illustrative application of the methodology.

Note that the scale for factor "closeness in time" does not relate to an absolute time scale (Figure 3). For example, the anchor point " 5 minutes" is meant to be an example of the concept of closeness in time, rather than a time measurement: a judgment of " 8 minutes" could be also placed on the " 5 minutes" anchor position if the analyst feels that the situations are not different. Alternative anchors for "closeness in time" are under evaluation, relating to typical tasks in NPP. In this way, as for example it was done in Kirwan (1997c), the judgement is not only connected with the numeric time separation.

Figure 8 shows the results of the expert elicitation of the input factors and of the anchors. The Figure show s the input interface of the model as seen by the analyst.

\section{Insert Figure 7. Influence diagram of the working model.}

Insert Figure 8. Results from elicitation of the input factors and the anchors: the dependence model as seen by the analyst. 


\subsection{Relationship between the input factors and dependence level}

The expert knowledge on dependence is converted into the FES rules. This is done by: 1) using statements given by the experts to fill some of the rules of the fuzzy expert system, and 2) filling the rest of the fuzzy rules via a rules interpolation approach.

The statements from the expert can be of different forms. In this paper each statement evaluates particular combinations of the input labels. When the expert is asked to evaluate the selected contexts, he/ she is also shown the relative positioning of the anchors on the scale, so as to help him/ her to contextualize his/ her statements (Figure 9). These evaluations allow partially filling the Table of rules, as shown in Figure 9 for "task relatedness".

Then, the missing "relationships" are filled in by an automatic "interpolation" procedure which smoothly spreads the consequent labels over the fuzzy rules (Marseguerra et al., 2004; Zio et al., 2009) (Figure 10).

Insert Figure 9. Table of rules for the intermediate variable Task relatedness (partial fill from the expert statements)

Insert Figure 10. Table of rules for the intermediate variable Task relatedness (complete fill by rules interpolation)

\section{Use of the working model: dependence in operators' early response to ATWS}

\subsection{Scenario description}

The case study considered refers to a set of operator actions required to avoid excessive boron dilution in the reactor cooling system in case of an Anticipated Transient Without Scram (ATWS) scenario in a nuclear Boiling Water Reactor (BWR).

In the considered scenario, the operators have successfully initiated the Standby Liquid Control System (SLCS) to shut the reactor down. To facilitate the reactor shut down, the operators are directed by the procedures to increase the voiding by reducing the level in the reactor to the Top of Active Fuel (TAF). Additionally, they are required to inhibit the actuation of the Automatic Depressurization System (ADS), which is activated by the signal of low water level in the reactor, generated while lowering the reactor water level to TAF. In case of failure to inhibit the ADS, the reactor pressure would be automatically decreased and low pressure injection systems (Core Spray 
System, CSS), would be activated. The injected water could dilute the boron injected by the SLCS and the consequential failure to control reactivity. In case of failure to inhibit ADS actuation, the operators are called to control the level in the reactor using low pressure injection, tripping one of the CSS pumps and controlling the other pump.

The signal to activate the ADS is generated about 7 minutes after the event of failure to scram. At that point, the operators have about 15 minutes to take actions to limit the low pressure injection flow.

The pair of operator tasks involved in the dependence assessment are 1) inhibit the ADS and 2) control the reactor vessel level in order to prevent diluting boron concentration after failure to inhibit the ADS. Both actions are directed by the same emergency procedure.

\subsection{Five cases of analyst judgment}

At first, three judgment cases are presented, corresponding to different possible interpretations of the context by the analyst. The last two cases show how the model responds to the variation of one input from cases 2 and 3.

\section{Case 1 - input judgments as point values on anchors}

Figure 11 shows an example of this type of input on the model interface and Figure 12 show s the corresponding fuzzification of the judgments by means of trapezoidal MFs.

- "Time" (upper left corner of Figure 11 and Figure 12): as said in the scenario description of Section 5.1, the separation in time of the two actions is expected to be around 15 minutes. The most reasonable anchor representing this context is " 5 minutes", the conservative judgment closest to the real context.

- "Cues" (upper right corner of Figure 11 and Figure 12): the initial cues for ADS inhibition are related to high reactor power level due to the failure to scram. The cues for control of low pressure injection are related to the reactor vessel level, which has to be manually maintained. This situation matches the anchor "different indicators for different parameters".

- "Goals" (lower right corner of Figure 11 and Figure 12): the two actions relate to the same function (shut down the reactor by boron control), carried out via different systems. This situation matches the anchor "same function by different systems".

- "Performers" (lower left corner of Figure 11 and Figure 12): the action is carried out by the same team. This matches the anchor "same team". 
Figure 13 reports the output of the dependence model as it results from the above judgments. The model assigns a level of "Low", without uncertainty (in the Figure, the possibility of "Low" is 1, while the possibility of all the other levels is 0 ). Without entering into the details of the underlying fuzzy expert system, the reason for this result is that the input judgments are such that only one rule is activated, i.e.:

IF Time is Close AND

THEN Dependence is Low

Cue similarity is None AND

Similarity of functions/ goals is High AND

Similarity of performers is High

Yet the analyst may not be satisfied with the judgments of Figure 11, as they do not incorporate entirely his/her belief on the context. In particular, ambiguity and uncertainty may come with his judgments. The next cases show how the presented model handles these aspects.

\section{INSERT Figure 11. Analyst input on the anchored scale for case 1: point estimates matching the anchors}

\section{INSERT Figure 12. Fuzzy input with trapezoidal MFs for case 1: point estimates matching the anchors.}

\section{INSERT Figure 13. Output of dependence level for case 1: point estimates matching the anchors.}

\section{Case 2 - input judgments as point values between labels or between anchors}

This case shows how the model responds to a judgment of the analyst which is in between labels. To better show the effect of this type of judgment, only one input is given as an "in between" judgment (i.e. similarity of cues), while the other ones are left to the values of Case 1 .

Consider the assessment on "cues" at the basis of the judgment in Case 1. An analyst may want to represent that cues are not totally different as it is implied in the description of Case 1. Indeed, ADS actuation is commanded by the signal of low water level in the reactor. Therefore, level in the reactor is also one of the parameters that the 
operators have to monitor while inhibiting ADS. The context is therefore more ambiguous than that represented in Case 1. The analyst may therefore feel more confident to assign a point assessment somewhere in between the label "NONE", representing "different indicators for different parameters" and the label "LOW", representing a somewhat higher level of depend ence.

Figure 14 shows the input of the analyst on the model interface and Figure 15 shows the corresponding fuzzification of the judgments by means of trapezoidal MFs.

Figure 16 reports the output of the dependence model in form of the possibility values of the different levels of dependence. It can be seen that the ambiguity of the judgment reflects in the model output, which assigns possibility to both levels of "low" and "medium" (in particular, 0.8 to LOW and 0.2 to MEDIUM). The relative possibilities values of the low and medium labels depend on the location of the input assessment arrow of the analysis in Figure 14.

The reason for this output is that as a result of the input judgments, two rules are activated, i.e.:

IF Time is Close AND

THEN Dependence is Low

Cue similarity is None AND

Similarity of functions/ goals is High AND

Similarity of performers is High

IF Time is Close AND

Cue similarity is Low AND

THEN Dependence is

Similarity of functions/ goals is High AND

MEDIUM

Similarity of performers is High

INSERT Figure 14. Analyst input on the anchored scale for case 2: point estimates between anchors.

INSERT Figure 15. Fuzzy input with trapezoidal MFs for case 2: point estimates between anchors.

INSERT Figure 16. Output of dependence level for case 2: point estimates between anchors.

Case 3 - range assessments (uncertainty) 
This case shows how uncertainty in the context can be represented in the judgment and how this reflects in the output dependence assessment. Again, only one input is varied from the judgments at the basis of Case 2 to show this effect.

Consider the judgment on the input factor "closeness in time". According to the scenario description, the operators have about 15 minutes to take actions to limit the low pressure injection flow. In Case 1, in order to have the input matching an anchor, the conservative judgment of 5 minutes was made. However, more realistically an analyst may want to represent the uncertainty in the "about 15 minutes" as an interval range between 5 and 20 minutes (Figure 17 and Figure 18).

Figure 19 reports the output of the dependence model in form of the possibility values of the different levels of dependence. It can be seen that as a consequence of the varied input judgment, the output assessment is spread on the three values of ZERO, LOW, and MEDIUM, peaked on the LOW level. The activated rules are in fact:

IF

Time is Neither AND

THEN Dependence is Zero

Cue similarity is None AND

Similarity of functions/ goals is High AND

Similarity of performers is High

IF $\quad$ Time is Neither AND

THEN Dependence is Low

Cue similarity is Low AND

Similarity of functions/ goals is High AND

Similarity of performers is High

IF Time is Close AND

THEN Dependence is Low

Cue similarity is None AND

Similarity of functions/ goals is High AND

Similarity of performers is High

IF Time is Close AND

THEN Dependence is

Cue similarity is Low AND

Medium

Similarity of functions/ goals is High AND

Similarity of performers is High

Insert Figure 17. Analyst input on the anchored scale for case 3: range assessment 


\title{
Insert Figure 18. Fuzzy input with trapezoidal MFs for case 3: range assessment.
}

\author{
Insert Figure 19. Output of dependence level for case 3: range \\ assessment.
}

\section{Cases 4 and 5 - response of the model to judgment variation on one input}

This cases show how the model responds to the variation of one input from the values that were used in cases 2 and 3. Note, these last two cases do not correspond to a specific dependence analysis assessment, but they are presented here as examples of sensitivity analysis.

The inputs for cases 4 and 5 are the same as those of cases 2 and 3, respectively, except for the judgment on factor "similarity of cues", which is now centered on the anchor "single indicator for the same parameter" (with falls on the in put label HIGH, without overlapping to any other label).

The output of the dependence model is reported in Figure 20: as expected, both output assessments are shifted tow ards higher dependence levels, as a consequence that the input judgment has been moved tow ards higher similarity of cues. In case 4, the level of HIGH is assigned without uncertainty (Figure 20, left), because the input assessment is such to activate only one rule:

IF Time is Close AND

THEN Dependence is

Cue similarity is High AND

High

Similarity of functions/ goals is High AND

Similarity of performers is High

Instead, the output distribution of case 5 is spread over two levels (MEDIUM and HIGH), as an effect of the uncertainty in the "closeness in time" judgment, which is expressed as an interval range (Figure 20, right). For brevity, the rules activated in case 5 are not reported.

Insert Figure 20. Output of dependence level for cases 4 (left) and 5 (right). 


\section{Discussion}

\subsection{Repeatability and traceability}

The motivation behind the development of the presented method has been to give repeatability and traceability to the dependence assessment among successive human actions affecting safety of installation.

Repeatability comes from the fact that the proposed method is based on an explicit, computable model. Indeed, as with DTs, the judgments are given on the input factors and the applicable dependence level follows from the model and not from direct expert judgment. With respect to the judgments to be provided on the input factors, repeatability benefits from providing anchor situations to the analyst: the more representative and defined the anchors are, the less controversial, and therefore variable, the input judgments are.

Unlike DTs, traceability of the dependence model is assured by a systematic expert elicitation approach, made up of two traceable steps (first fill and interpolation of the fuzzy logic rules relating the input to the dependence level). Once the dependence model is built, it is easy to go back and verify the base expert's statements that originated the model. In principle, the traceability of the process to build DTs could be potentially improved by providing better documentation, but this has been rarely the case.

\subsection{Verification and validation issues}

Model verification and validation are two essential steps in the development of any method and are being taken into consideration in the development of the present dependence method.

Verification is intended as the process to make sure that the model represents correctly the experts' knowledge (O'Keefe \& Smith, 1987). After construction, the expert model has to be assessed (verified) by the experts whose expertise is captured in the model. In this phase, feedback needs to be provided to the experts to allow them to determine whether the expert system model is a good representation of their knowledge, and if not, to modify the model appropriately. Techniques for providing this feedback are currently under investigation by the authors: they include visualization techniques as well as importance measures. Some preliminary results can be found in Podofillini et al. (2008).

A fundamental step for the acceptance of the dependence model is its validation. Yet, the empirical validation of a human error dependence model is a very difficult task. In what follows, the status of HRA validation generally and as it regards dependence is 
outlined. The most significant HRA validation efforts have addressed mostly failure probabilities for "execution", that is, carrying out a series of actions or steps on a system. A review of validation efforts for a number of HRA methods can be found in Kirwan (1997b). These sources note the difficulty of validating the failure probabilities for decision or diagnosis that are predicted with HRA methods. This is due in particular to the lack of reference data, which in turn is caused by the sensitivity of decision failures to a broad range of variables (contextual factors). Indeed, data collection efforts have addressed mostly failure probabilities for "execution", as, for example, in Kirwan, et al. (2008), where the focus is on communication errors.

There are on-going efforts that should improve the state of HRA data in the future. One of these is an attempt to analyze operating experience and to obtain in this way empirical relationships between the factors and the observed human failure events: the Human Error Repository and Analysis (HERA) project and database sponsored by the U.S. Nuclear Regulatory Commission (Hallbert et al., 2006). A second effort is the International HRA Empirical Study being performed by an international group of organizations jointly with the OECD Halden Reactor Project, in which the predictions of HRA methods are being compared with simulator data (Lois et al., 2008 and Dang et al., 2007). While both quantitative predictions (the HEPs) and qualitative predictions (the "driving" or most important input factors identified in the Human Reliability Analysis) will be addressed in this work, the number of data points will not be sufficient to validate comprehen sively the relation ship between the input variables and the predicted failure probabilities, a relationship represented by each HRA method.

Concerning the validation of a dependence model, the basis or mechanisms that potentially lead to dependence within a series of actions relate strongly to the decisionmaking of the personnel associated with these actions. Therefore, one may anticipate that data will be very difficult to collect. A major reason is that the action and failure probability of interest are conditioned on a previous personnel failure. Given the expected performance levels, this initial failure is relatively difficult to "provoke" systematically and realistically such that the subsequent performance can be examined. In case the validation strategy would be to test the model's predictions against simulator data, this poses challenges also as to how the simulator experiments should be designed (e.g. what accident scenarios should be simulated).

This suggests that a model of dependence for HRA cannot be based on (built from) a set of data from which the overall relationship between the input variables and the output variable of interest can be quantitatively estimated. For the same reason, it can be expected that the validation of such a model cannot be done against a 
comprehensive set of $d$ ata that is able to explore extensively the range of the model response.

However, these difficulties should not discourage and some way of testing the empirical basis of the model should be pursued. Three concept alternatives can be anticipated here:

- Validation of the model against a limited set of data, thus validating only some of the input-output relationships, only those that cover the considered data.

- Validation of the effect of individual factors. This would address the question if I how the effect of variations in one input factor (averaging the effect of the other factors or keeping these at fixed values) as anticipated by the model compares with the empirical data.

- Validation of the relative strength of the factors and of their interactions. This would address the question if the factors that are predicted as being important by the model result as being important also from the data.

It is expected that the experience with the mentioned International HRA Empirical Study will help in defining how to go about the above concept alternatives.

While validation against empirical data has the mentioned challenges, alternative approaches to validation are being considered for the short term.

Indeed, there is a significant history working with the THERP dependence approach, in its original formulation or supported by DTs. This can be used to draw some conclusions on the reasonableness of the numbers produced for dependence calculations, i.e. on its so-called face validity (Kirwan, 1997a).

Data from experts can also be used to replace empirical data for a validation exercise. This can be done in two ways (which are not exclusive). The first is to give case studies to the experts and partition the cases in two sets: one set is used to inform the relationships and build the model and the rest is used to test the prediction capability of the model. The other way is to test the predictions against those from another set of experts. These options will be considered in the design of the expert elicitation process.

\subsection{Additional discussion}

It is worth noting that other expert modelling approaches exist that can handle dependence among the input factors. Probabilistic models such as influence diagrams and Bayesian belief networks (Phillips et al., 1990) and connectionism networks 
(Sträder, 2000) are some examples. Research is also being performed by the authors to compare the performance of these probabilistic approaches with Fuzzy approaches.

Finally, note that the so-called second-generation HRA methods (ATHEANA (Cooper et al., 1996), MERMOS (Le Bot et al., 1998), CREAM (Hollnagel, 1998)) do not quantify dependence based on conditional HFEs probabilities as the presented method assumes. Their common notion is that the likelihood of HFEs is driven by performance conditions determined by the context where the action takes place, rather than by intrinsic human error probabilities associated with the task. Following this notion, the context must include preceding HFEs and the failure probability estimated for any action should reflect a) the effect of preceding HFEs on the scenario and on the operators' situation assessment, and b) the relationships between the actions, which would include many of the dependence factors. In this way, a model of dependence remains essential in a second-generation analysis even if the proposed dependence assessment method may not be applicable. As second-generation methods have not yet been extensively applied, improving dependence assessment and quantifying conditional probabilities remain issues of major concern.

Another way of handling dependence is through the use of dynamic PSA tools, see e.g. (Chang \& Mosleh, 2007), which allow to directly simulate the evolution of the system after each human intervention and therefore, in principle, to better define the context in which the dependent actions are carried out.

\section{Conclusions and Outlook}

Human failure dependence assessment is a highly subjective part of HRA and efforts to improve the transparency and repeatability of the assessments are needed. This paper proposes a dependence method that is based on an expert model, built from a transparent expert elicitation process. The expert model is a Fuzzy Expert system. This representation has been selected since it is suitable for models mostly built from expert judgment, as opposed to empirical data. A working model of dependence has been developed to investigate the concepts underlying the proposed method; its internal relationships have been set by the authors. The expert elicitation exercise will be performed in the future. The model has been applied for dependence assessment of two operator actions in response to an anticipated transient without scram in a nuclear boiling water reactor.

Improving the transparency and repeatability of human reliability dependence assessment, while keeping the method practical to use have been the goals of the research. The features of the model to achieve these goals are as follows. 
The proposed method is based on an explicit, computable model. As in a decision tree, an analyst is required to give judgments on the input factors of the model, and not directly on the dependence level (which is the output of the model). While improving the repeatability of the assessment, this is also expected to reduce its uncertainty, compared to the case of giving judgments directly on the dependence level. Indeed, uncertainty on input judgments exists as well, but with anchors to provide references for the scale, the inputs can be made less subjective than the dependence level.

The computable model is built from a systematic expert elicitation approach, made up of two traceable steps. This is important because, in connection with what said above, the accuracy of the results produced by the method depends on the accuracy of the computable model. Given that empirical validation of the model accuracy is extremely challenging, it is important that the building process is traceable, to allow scrutinizing the experts' statements contained in the expert model.

With the use of the expert model, the analyst's input judgments are directly and formally converted into the output, the (discrete) dependence level. Compared to a decision tree-based approach, the result gives a dependence assessment that can more closely reflect the analysts' understanding of the dependence factors for a given set of tasks.

Anchor situations are provided as guidance for the analyst's assessment of the input factors. Using up to five input linguistic labels for the factors, compared to the typical two of (binary) decision trees, may be perceived as subject to more subjectivity as well as cumbersome by analysts and experts. However, the anchor points may counteract the effect of having more labels for each factor, by providing concrete references for determining the input factor for the specific case under analysis.

Neither the HRA analysts nor the experts whose knowledge is represented by the expert model need to be familiar with Fuzzy Expert systems: no aspect of the Fuzzy formalism is shown to analysts and experts and they interface only with the input linguistic labels and with the anchor points. This is very similar to the interface of decision trees commonly used (e.g. SPAR-H and the EPRI HRA Calculator ${ }^{\circledR}$ ).

Once the model is finally built, verification and validation are also important aspects. In particular, validation of the dependence model against empirical data is significantly challenging, due to the difficulty of collecting failure probability data. However, it is expected that it will be possible to draw some conclusions on the model face validity. Indeed, although the currently used THERP-based approaches may suffer from a number of limitations, there is a significant experience in working with these 
and this can be used to check the reasonableness of the results produced by the proposed method.

\section{References}

American Society of Mechanical Engineers, ASME Standard for Probabilistic Risk Assessment for Nuclear Power Plant Applications, ASME RA-S-2002 (with addenda, RA-Sb-2005), New York: ASME, 2005.

Brewer, N. T. \& Chapman, G. B. (2002). The fragile basic anchoring effect. Journal of Behavioral Decision Making, 7, 223-242.

Cepin, M. (2006). Development of a method for consideration of dependence between human failure events. Proceedings of ESREL 2006 - Safety and Reliability for managing risk, Estoril, Portugal, 18-22 September 2006.

Cepin, M. (2008a). DEPEND-HRA - A method for consideration of dependency in human reliability analysis. Reliability Engineering \& System Safety, 93(10), 14521460.

Cepin, M. (2008b). Importance of human contribution within the human reliability analysis (IJS-HRA). Journal on loss prevention in process industries, 21(3), 268276.

Cepin, M. (2008c). Comparison of methods for dependency determination between human failure events within human reliability analysis, Science and technology of nuclear installations, Vol. 2008, 1-7.

Chang, S.H., Kang, K.S., Choi, S.S., Jeong, H.K., \& Yi, C.U. (1995). Development of the Online Operator Aid System Oasys Using a Rule-Based Expert-System and Fuzzy-Logic for Nuclear-Power-Plants. Nuclear Technology 112 (2), 266-294.

Chang, Y.H.J., Mosleh, A. (2007). Cognitive modeling and dynamic probabilistic simulation of operating crew response to complex system accidents: Part 1: Overview of the IDAC Model. Reliability Engineering \& System Safety, 92(8), 997-1013.

Cheok, M.C., Parry, G. W. \& Sherry, R. R. (1998). Use of importance measures in riskinformed regulatory applications. Reliability Engineering \& System Safety, 60, 213-26.

Cooke, R.M. (1991). Experts in uncertainty, New York: Oxford university press.

Cooper, S. E., Ramey-Smith, A. M., Wreathall, J., Parry, G.W., Bley, D.C., Luckas, W.J., Taylor, H.J., \& Barriere, M.T. (1996). A Technique for Human Error Analysis (ATHEANA). NUREG/CR-6350. Washington, DC: U.S. Nuclear Regulatory Commission.

Dang, V.N., Bye, A., Lois, E., Forester, J., Kolaczowski, A.M. \& Braarud, P.O. (2007) An Empirical Study of HRA Methods - Overall Design and Issues, Proc. 2007 IEEE 8th Human Factors and Power Plants (HFPP) and 13th Annual HPRCT Meeting, August 26-31, 2007, Monterey, CA, USA.

Gertman, D., Blackman, H., Marble, J., Byers, J., Haney, L., \& Smith, C. (2005). The SPAR-H Human Reliability Analysis Method. NUREG/CR-6883. Washington, DC: US Nuclear Regulatory Commission.

Hollnagel, E. (1998) Cognitive reliability and error analysis method (CREAM). Oxford: Elsevier Science Ltd. 
Huang, D., Chen, T., \& Wang, M.J.J. (2001). A fuzzy set approach for event tree analysis. Fuzzy Sets and Systems 118(1), 153-165.

Grobbelaar, J., Julius, J. \& Rahn, F. (2005). Analysis of Dependent Human Failure Events Using the EPRI HRA Calculator. Proc. of the ANS Topical Meeting on Probabilistic Safety Assessment (PSA'05), San Francisco, CA, USA, Sept 11-15, 2005.

Kawamoto, K., Houlihan, C.A., Balas, E.A., \& Lobach, D.F. (2005). Improving clinical practice using clinical decision support systems: a systematic review of trials to identify features critical to success. British Medical Journal (BMJ), doi:10.1136/bmj.38398.500764.8F (published 14 March 2005).

Kennedy, G.A.L., Siemieniuch, C.E., Sinclair, M.A, Kirwan, B.A. \& Gibson, H.W. (2007). Proposal for a sustainable framework process for the generation, validation, and application of human reliability assessment within the engineering design lifecycle. Reliability Engineering \& System Safety, 92(6), 755-770.

Kim, B., \& Bishu, R.R. (1996). On assessing operator response time in human reliability analysis (HRA) using a possibilistic fuzzy regression model. Reliability Engineering \& System Safety 52(1), 27-34.

Kirwan, B. (1997a). Validation of human reliability assessment techniques: part 1 validation issues. Safety Science 27(1), 25-41.

Kirwan, B. (1997b). Validation of human reliability assessment techniques: part 2 validation results. Safety Science 27(1), 43-75.

Kirwan, B. (1997c). The development of a nuclear chemical plant human reliability management approach: HRMS and JEDI. Reliability Engineering and System Safety 56, 107-133.

Kirwan, B., Gibson, W. H. \& Hickling, B. (2008). Human error data collection as a precursor to the development of a human reliability assessment capability in air traffic management. Reliability Engineering \& System Safety, 93(2), 217-233.

Kirwan, B., Umbers, I., Edmunds, J. and Gibson, H. (2008). Quantifying the Unimaginable - the Case for Human Performance Limiting Values. Proc. of the 9th Int. Conf. on Probabilistic Safety Assessment and Management (PSAM9), Hong Kong, China, 18-23 May 2008.

Konstandinidou, M., Nivolianitou, Z., Kiranoudis, C., \& Markatos, N. (2006). A fuzzy modeling application of CREAM methodology for human reliability analysis. Reliability Engineering and System Safety 91(6), 706-716.

Hallbert, B., Boring, R., Gertman, D., Dudenhoeffer, D., Whaley, A., Marble, J.,Joe, J., Lois, E. (2006), Human Event Repository and Analysis (HERA) System, Overview, NUREG/CR-6903, Vol. 1 Washington, DC: US Nuclear Regulatory Commission.

Le Bot, P., Desmares, E., Bieder, C., Cara, F., \& Bonnet, J-L. (1998). MERMOS: unprojet d'EDF pour la mise à jour de la méthodologie EPFH. Revue Generale Nucleaire, 1998, 1.

Liang, G.S., \& Wang, M.J.J. (1993). Evaluating human reliability using fuzzy relation. Microelectronics and Reliability, 33(1), 63-80.

Lois, E., Dang, V.N., Forester, J., Broberg, H., Massaiu, S., Hildebrandt, M., Braarud, P.O., Parry, G., Julius, J., Boring, R.L., Männistö, I., Bye, A. (2008). International HRA Empirical Study - Description of Overall Approach and First Pilot Results 
from Comparing HRA Methods to Simulator Data, Halden Reactor Project Work Report HWR-844, Apr. 2008.

Marseguerra, M., Zio, E., \& Bianchi, M. (2004). A fuzzy modelling approach to road transport with application to a case of spent nuclear fuel transport. Nuclear Technology 146(3), 290-302.

Marseguerra, M., Zio, E. \& Librizzi, M. (2006). Quantitative Developments in the Cognitive Reliability and Error Analysis Method (CREAM) for the Assessment of Human Performance. Annals of Nuclear Energy, 33(10), 894-910.

O'Hagan, A., Buck, C. H., Daneshkhah, A., Eiser, J. R., Garthwaite, P. H., Jenkinson, D. J., Oakley, J. E., Rakow, T. (2006). Uncertain Judgments: Eliciting Experts' Probabilities, Chichester, England: John Wiley \& Sons.

O'Keefe, R.M., Smith, E.P. (1987). "Validating Expert System Performance". IEEE Expert, 2, pp. 81-89.

Onisawa, T. (1988a). Fuzzy concepts in human reliability. In: Fuzzy Logic in Knowledge-Based Systems, Decision and Control. North-Holland, New York: M. M. Gupta, T. Yamakawa.

Onisawa, T. (1988b). A representation of human reliability using fuzzy concepts. Information Sciences 45 (2), 153-173.

Otway, H., von Winterfeldt, D. (1992). Expert Judgment in Risk Analysis and Management: Process, Context, and Pitfalls. Risk Analysis, 12, 83-93.

Phillips, L. D., Humphreys, P., Embrey, D. E. \& Selby, D. L. (1990). A Socio-Technical Approach to Assessing Human Reliability. In: Influence Diagrams, Belief Nets and Decision Analysis, New York: R. M. Oliver \& J. Q. Smith. Wiley.

Podofillini, L., Dang, V.N., Zio, E., Baraldi, P. \& Librizzi, M. (2008). Techniques for verification of expert models for dependence assessment in human reliability analysis. Proc. of the 9th Int. Conf. on Probabilistic Safety Assessment and Management (PSAM9), Hong Kong, China, 18-23 May 2008.

Richei, A., Hauptmanns, U., \& Unger H. (2001). The human error rate assessment and optimizing system HEROS - a new procedure for evaluating and optimizing the man-machine interface in PSA. Reliability Engineering \& System Safety 72 (2), 153-164.

Suresh, P.V., Babar, A.K., \& Raj, V.V. (1996). Uncertainty in fault tree analysis: A fuzzy approach. Fuzzy Sets and Systems 83, 135-141.

Swain, A.D., \& Guttman, H.E. (1983). Handbook of human reliability analysis with emphasis on nuclear power plant applications. NUREG/CR-1278. Washington, DC: US Nuclear Regulatory Commission.

Stevens, S. S. (1946). On the Theory of Scales of Measurement. Science, 103, 677-680.

Sträter, O. (2000). Evaluation of the human reliability based on human experience. $\mathrm{PhD}$ dissertation of Oliver Sträter, GRS-107, GRS - Gesellschaft für Anlagen- und Reaktorsicherheit.

Terano, T., Murayama, Y., \& Akiyama, N. (1983). Human reliability and safety evaluation of man-machine systems. Automatica, 19 (6), 719-722.

US Nuclear Regulatory Commission (2005). Good Practices for Implementing Human Reliability Analysis, NUREG-1792, US Nuclear Regulatory Commission, Washington DC, USA. 
Vaurio, J.K. (2001). Modelling and quantification of dependent repeatable human errors in system analysis and risk assessment. Reliability Engineering and System Safety 71, 79-188.

Whaley, A.M., Boring, R. L., Blackman, H. S., McCabe, P. H. \& Hallbert, B. P. (2007). Lessons Learned from Dependency Usage in HERA: Implications for THERPRelated HRA Methods, In: Proc. of 8-th IEEE Conference on Human Factors and Power Plants, Monterrey, California, 26-31 August 2007.

Zadeh, L.A. (1965). Fuzzy sets. Inform. And Control 8, 338-353.

Zio, E., Baraldi, P., Librizzi, M., Podofillini, L. \& Dang, V.N. (2009). A Fuzzy Expert System for modeling dependence among human errors. Submitted to Fuzzy Sets and Systems. 
Table 1. The EPRI HRA Calculator @ dependence decision tree (Grobbelaar et al., 2005).

\begin{tabular}{|c|c|c|c|}
\hline Time between cues & $\begin{array}{l}\text { Adequate } \\
\text { resources }\end{array}$ & Stress & Level \\
\hline \multirow{4}{*}{ Simultaneous } & \multirow{2}{*}{ No } & High & \multirow{2}{*}{ CD } \\
\hline & & Low & \\
\hline & \multirow{2}{*}{ Yes } & High & $\mathrm{CD}$ \\
\hline & & Low & HD \\
\hline \multirow{2}{*}{$0-15 \min$} & \multirow{2}{*}{ Yes } & High & $\mathrm{CD}$ \\
\hline & & Low & HD \\
\hline \multirow{2}{*}{$15-30 \mathrm{~min}$} & \multirow{2}{*}{ Yes } & High & HD \\
\hline & & Low & MD \\
\hline \multirow{2}{*}{$30-60 \mathrm{~min}$} & \multirow{2}{*}{ Yes } & High & $\mathrm{MD}$ \\
\hline & & Low & LD \\
\hline \multirow{2}{*}{$>60 \mathrm{~min}$} & \multirow{2}{*}{ Yes } & High & LD \\
\hline & & Low & $\mathrm{ZD}$ \\
\hline
\end{tabular}

NOTE: The above table from the EPRI HRA Calculator ${ }^{\circledR}$ applies for two actions performed by the same crew, at the same location (the control room) and guided by different cues 
Table 2. Linguistic variables, qualitative labels and anchor points for the working model

\begin{tabular}{|c|c|c|}
\hline Linguistic variable & Qualitative labels & $\begin{array}{l}\text { Anchors }{ }^{1} \text { (provided by the } \\
\text { expert) }\end{array}$ \\
\hline \multirow[t]{6}{*}{ Closeness in time } & \multirow{6}{*}{$\begin{array}{l}\text { close in time, widely } \\
\text { separated in time, } \\
\text { neither }\end{array}$} & $24 \mathrm{~h}$ \\
\hline & & $8 \mathrm{~h}$ \\
\hline & & $1 \mathrm{~h}$ \\
\hline & & $30 \mathrm{~min}$ \\
\hline & & $20 \mathrm{~min}$ \\
\hline & & $5 \min$ \\
\hline \multirow[t]{5}{*}{ Cue similarity } & \multirow[t]{5}{*}{$\begin{array}{l}\text { None/very low, low, medium, } \\
\text { high, very high/complete }\end{array}$} & $\begin{array}{l}\text { Different sets of indicators for different } \\
\text { parameters }\end{array}$ \\
\hline & & $\begin{array}{l}\text { Different sets of indicators for the } \\
\text { same parameter }\end{array}$ \\
\hline & & Single indicator for the same parameter \\
\hline & & $\begin{array}{l}\text { Different sets of indicators for the } \\
\text { same physical quantity }\end{array}$ \\
\hline & & $\begin{array}{l}\text { Same sets of indicators for the same } \\
\text { sets of parameters }\end{array}$ \\
\hline \multirow[t]{4}{*}{ Function/goal similarity } & \multirow[t]{4}{*}{$\begin{array}{l}\text { None/very low, low, medium, } \\
\text { high, very high/complete }\end{array}$} & $\begin{array}{l}\text { Different functions by different } \\
\text { systems }\end{array}$ \\
\hline & & Different functions by the same system \\
\hline & & Same function by different systems \\
\hline & & Same function by the same system \\
\hline \multirow[t]{5}{*}{ Similarity of performers } & \multirow{5}{*}{$\begin{array}{l}\text { None/very low, low, medium, } \\
\text { high, very high/complete }\end{array}$} & TSC vs control room shift \\
\hline & & Different teams \\
\hline & & $\begin{array}{l}\text { Different individuals (same } \\
\text { qualification) }\end{array}$ \\
\hline & & Same team \\
\hline & & Same person \\
\hline
\end{tabular}

\footnotetext{
${ }^{1}$ It is expected that multiple sets of time anchors will be distinguished in the final model. For example, time anchors should be placed in different locations on the scale for short and long term actions: a time separation of 20 minutes between the two actions, can be considered as wide if the first action is to be performed few minutes after the annunciation of the related cues: on the other hand, the same separation of 20 minutes can be considered as "very small" if the first action is to be performed within hours from the annunciation of the related cues.
} 


\section{Dependency Condition Table}

\begin{tabular}{|c|c|c|c|c|c|c|}
\hline $\begin{array}{l}\text { Condition } \\
\text { number }\end{array}$ & $\begin{array}{l}\text { Crew } \\
\text { (same or } \\
\text { different) }\end{array}$ & $\begin{array}{c}\text { Time } \\
\text { (close in time } \\
\text { or not close in } \\
\text { time) }\end{array}$ & $\begin{array}{l}\text { Location } \\
\text { (same or } \\
\text { different) }\end{array}$ & $\begin{array}{c}\text { Cues } \\
\text { (additional } \\
\text { or no } \\
\text { additional) }\end{array}$ & Dependency & $\begin{array}{c}\text { Number of Human Action Failures Rule } \\
\square-\text { Not Applicable. } \\
\text { Why? }\end{array}$ \\
\hline 1 & \multirow[t]{8}{*}{$\mathrm{S}$} & \multirow[t]{4}{*}{$\mathrm{c}$} & \multirow[t]{2}{*}{$\mathrm{S}$} & na & Complete & \multirow{17}{*}{$\begin{array}{c}\text { When considering recovery in a series } \\
\text { e.g., } 2^{\text {nd }}, 3^{\text {rd }} \text {, or } 4^{\text {th }} \text { checker }\end{array}$} \\
\hline 2 & & & & $\mathrm{a}$ & Complete & \\
\hline 3 & & & \multirow[t]{2}{*}{ d } & na & High & \\
\hline 4 & & & & $\mathrm{a}$ & High & \\
\hline 5 & & \multirow[t]{4}{*}{ nc } & \multirow[t]{2}{*}{$\mathrm{s}$} & na & High & \\
\hline 6 & & & & $\mathrm{a}$ & Moderate & \\
\hline 7 & & & \multirow[t]{2}{*}{$\mathrm{d}$} & na & Moderate & \\
\hline 8 & & & & $\mathrm{a}$ & Low & \\
\hline 9 & \multirow[t]{8}{*}{$\mathrm{d}$} & \multirow[t]{4}{*}{$\mathrm{c}$} & \multirow[t]{2}{*}{$\mathrm{s}$} & na & Moderate & \\
\hline 10 & & & & $\mathrm{a}$ & Moderate & \\
\hline 11 & & & \multirow[t]{2}{*}{$\mathrm{d}$} & na & Moderate & \\
\hline 12 & & & & $\mathrm{a}$ & Moderate & \\
\hline 13 & & \multirow[t]{4}{*}{$\mathrm{nc}$} & \multirow[t]{2}{*}{$\mathrm{s}$} & na & Low & \\
\hline 14 & & & & $\mathrm{a}$ & Low & \\
\hline 15 & & & \multirow[t]{2}{*}{ d } & na & Low & \\
\hline 16 & & & & $\mathrm{a}$ & Low & \\
\hline 17 & & & & & Zero & \\
\hline
\end{tabular}

Figure 1. The SPAR-H dependence decision tree (Gertman et al., 2005).

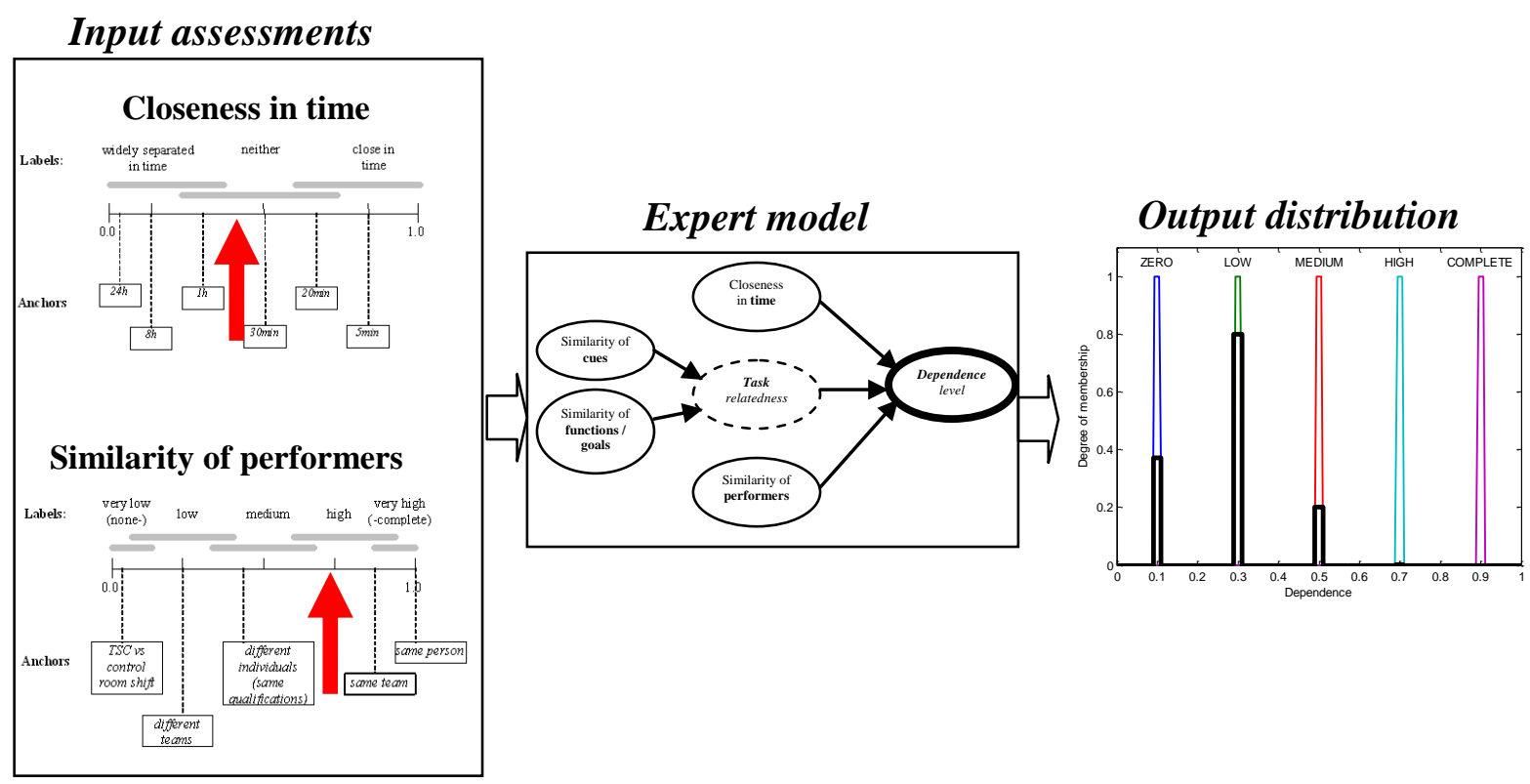

Figure 2. Overview of the dependence model. Different models are needed for preinitiators, and different types of post-initiators. 


\begin{tabular}{|c|c|c|}
\hline None & High & Very high \\
\hline &  & 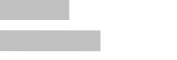 \\
\hline 0 & $\begin{array}{l}\text { Analyst } \\
\text { Input } 2\end{array}$ & ש \\
\hline & chor D & Anchor B \\
\hline
\end{tabular}

Figure 3. Analyst elicitation on anchored scale; two analyst input types are shown: as a point value (arrow) and as an interval (horizontal bar).
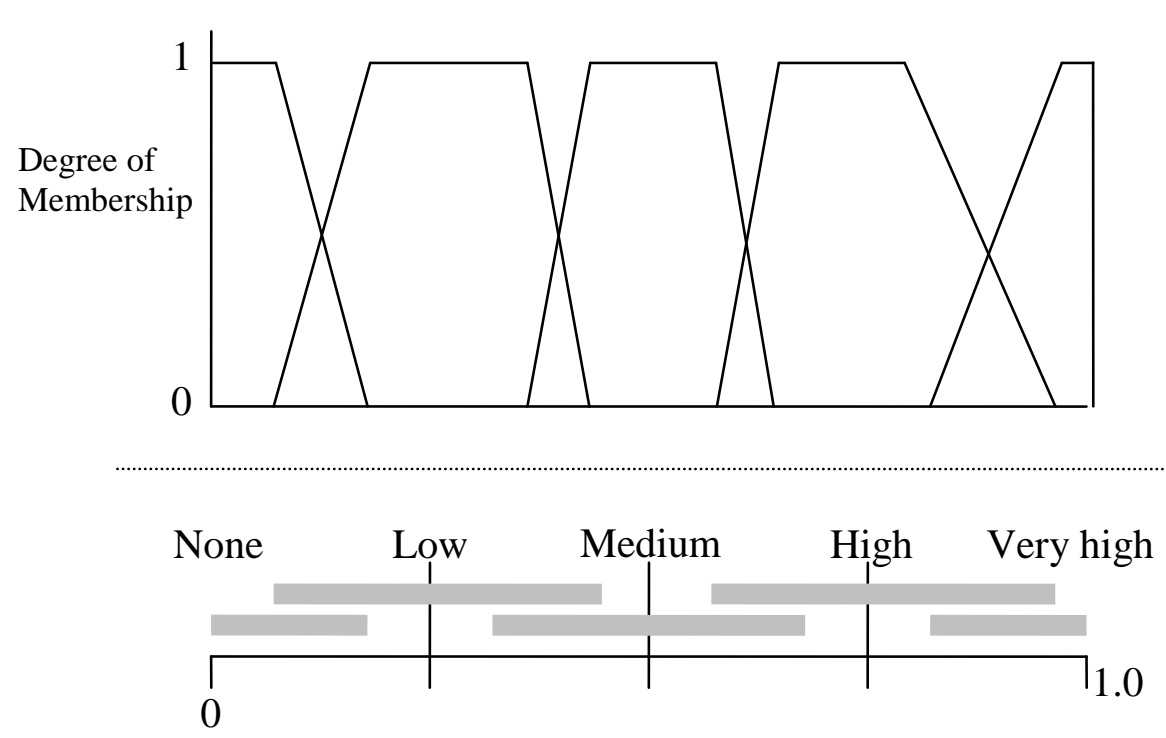

Figure 4. Possible association of fuzzy sets and membership functions (trapezoids) to the input qualitative variable. 

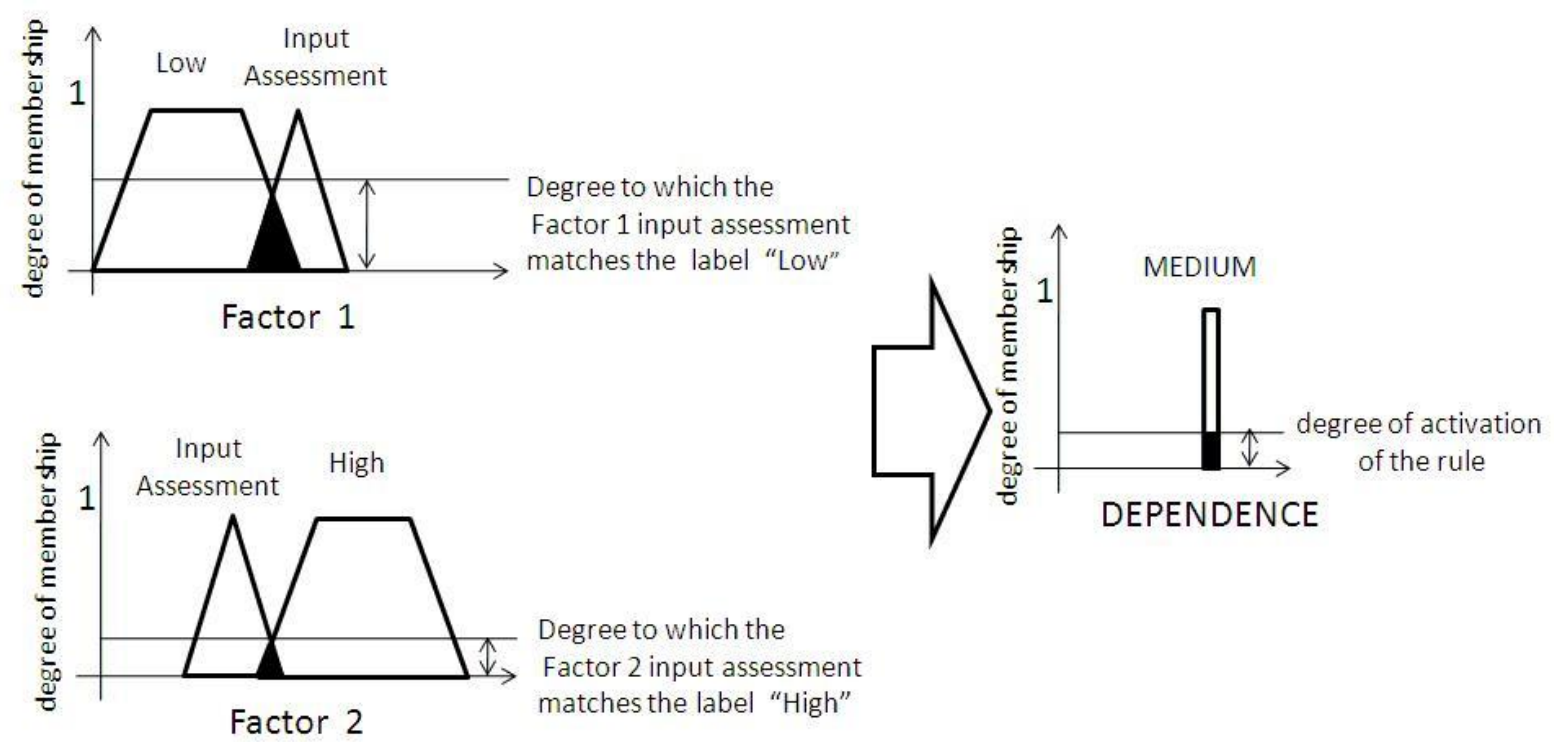

Figure 5. Left: example of computation of the degree to which the Factor 1 input assessment matches the label "Low" and the Factor 2 input assessment the label "High" . Right: computation of the degree of activation of the rule If "Factor 1 " is "Low" and "Factor 2" is "High" THEN "Dependence" is "Medium" as minimum value between the degrees to which Factor 1 and Factor 2 input assessments match the corresponding labels in the rule.

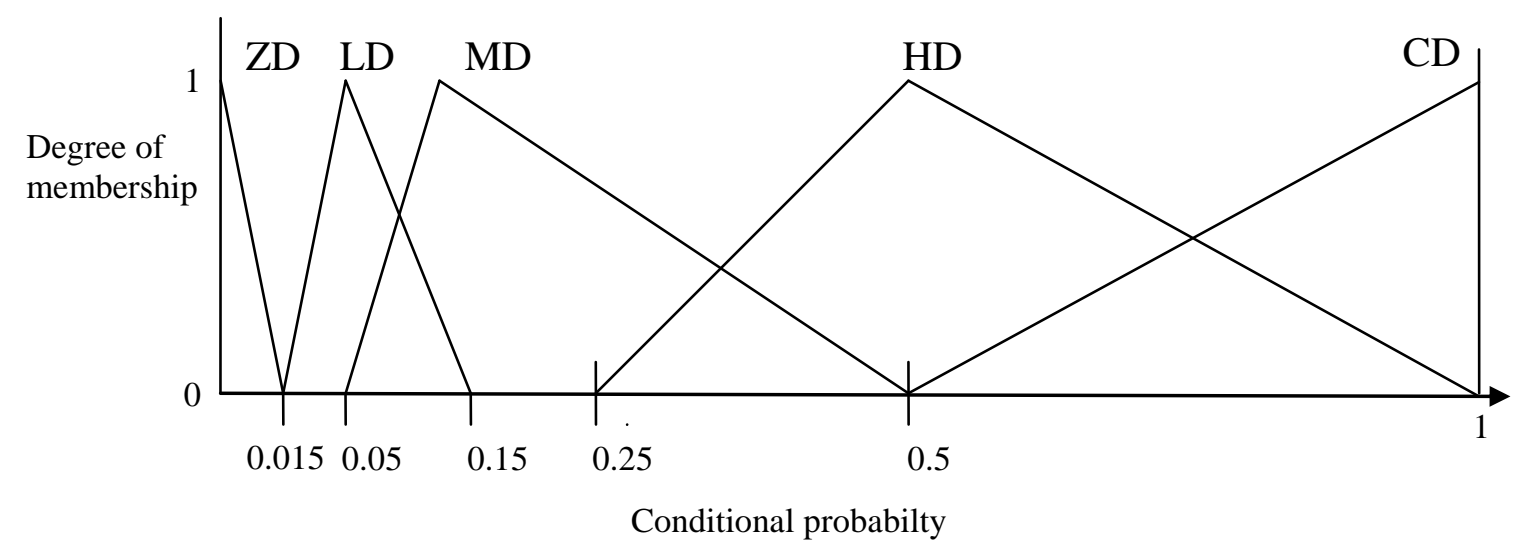

Figure 6. Fuzzy sets and membership functions associated to the THERP dependence levels. Elicited from THERP guidelines (Swain \& Guttman, 1983). 


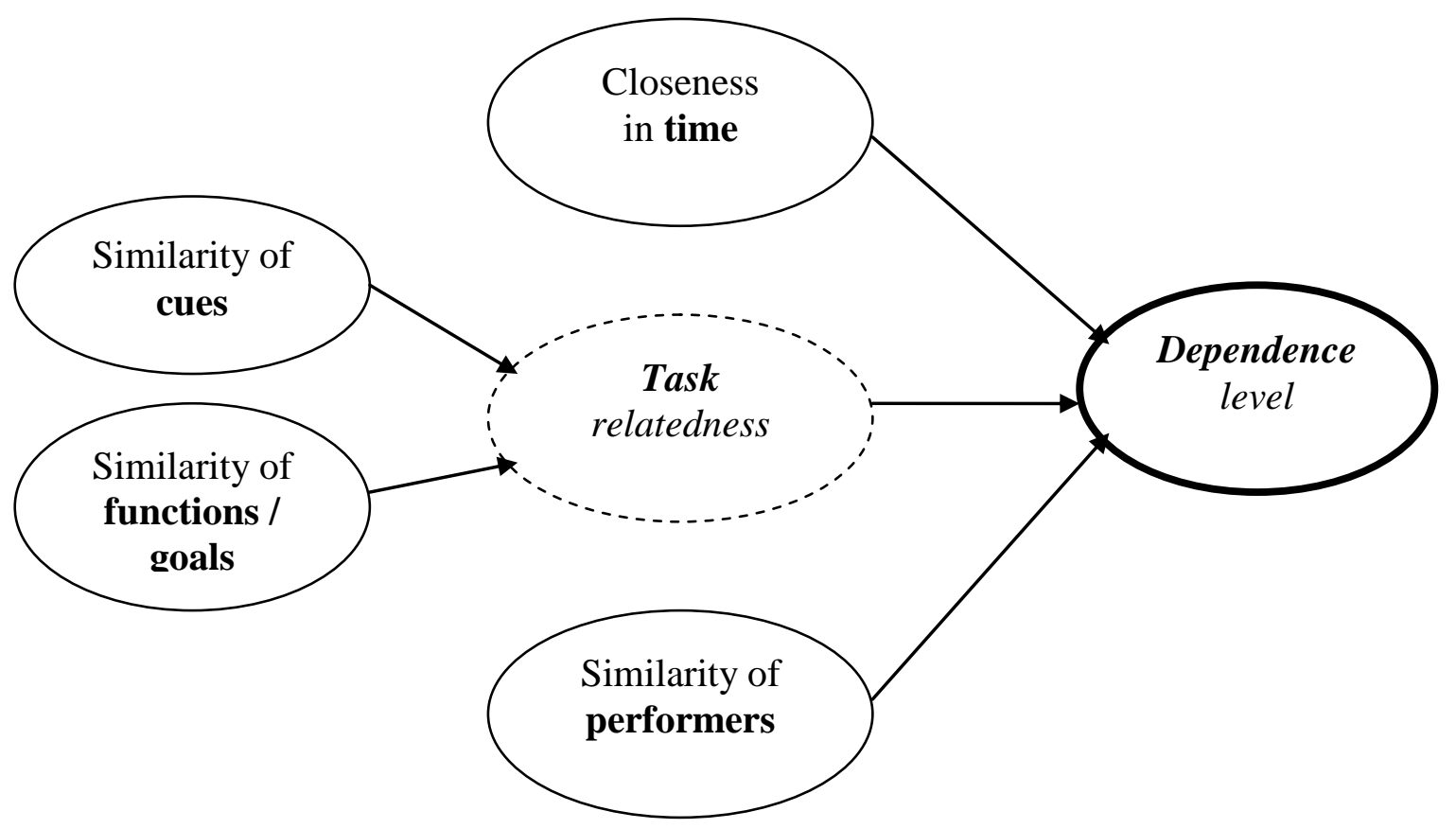

Figure 7. Influence diagram of the working model. 


\section{Closeness in time}

Labels:

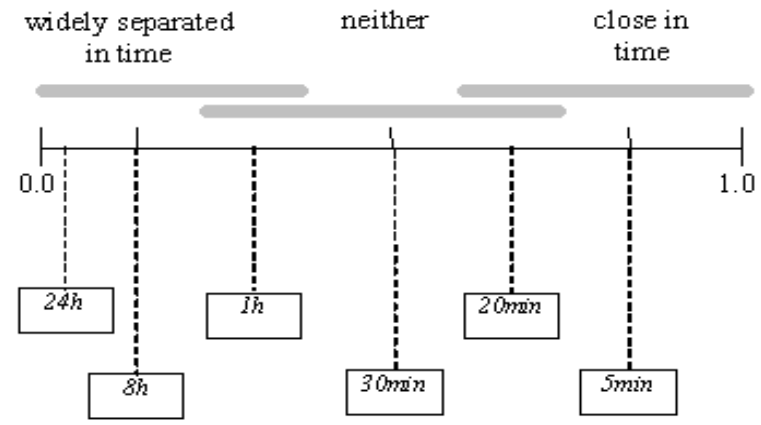

Similarity of functions/goals

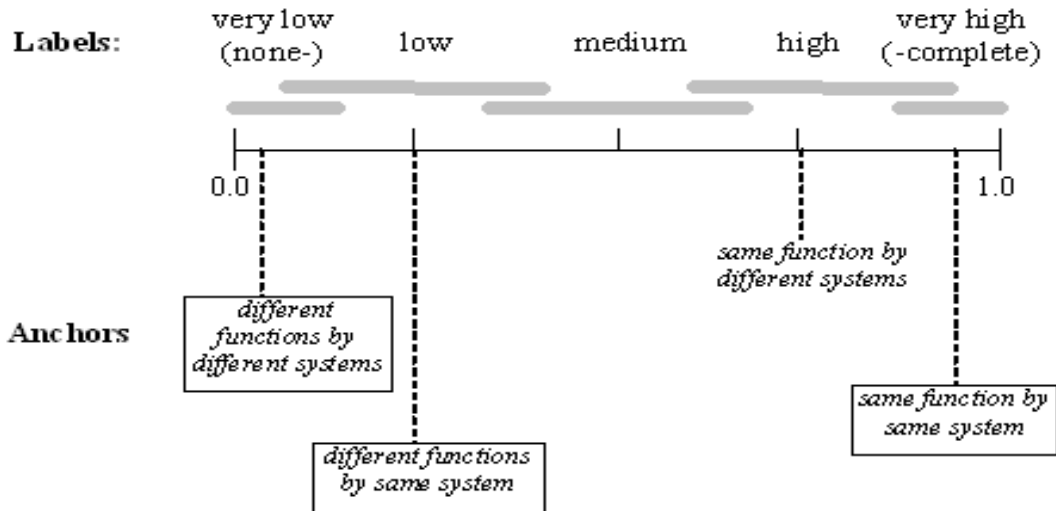

\section{Similarity of performers}

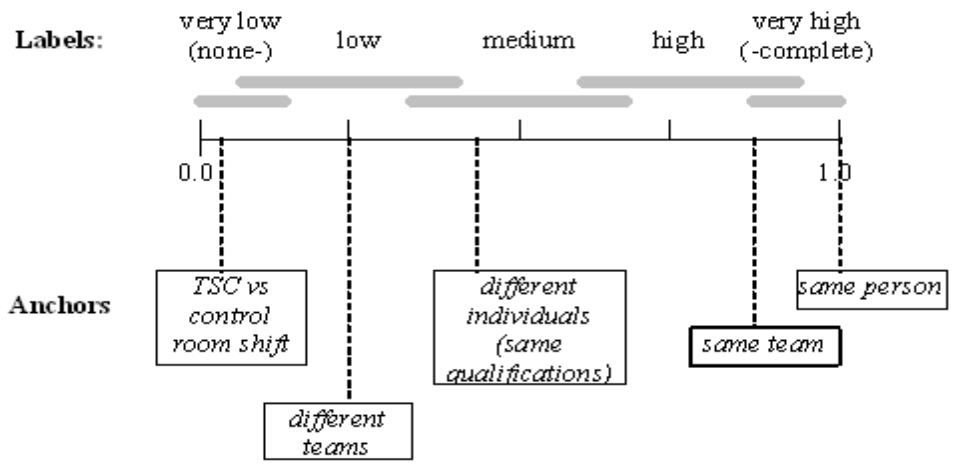

Similarity of cues

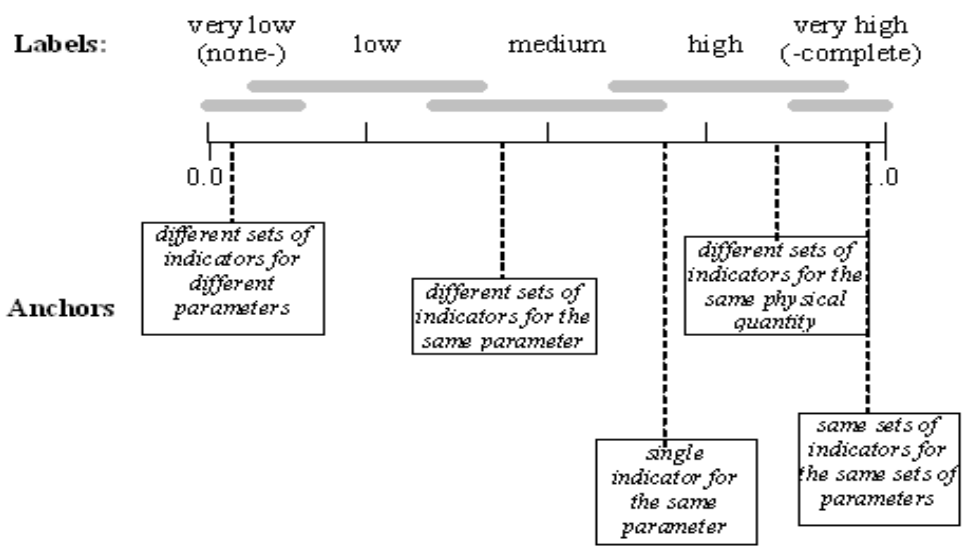

Figure 8. Results from elicitation of the input factors and the anchors: the dependence model as seen by the analyst. Anchors may change for different typologies of tasks. 


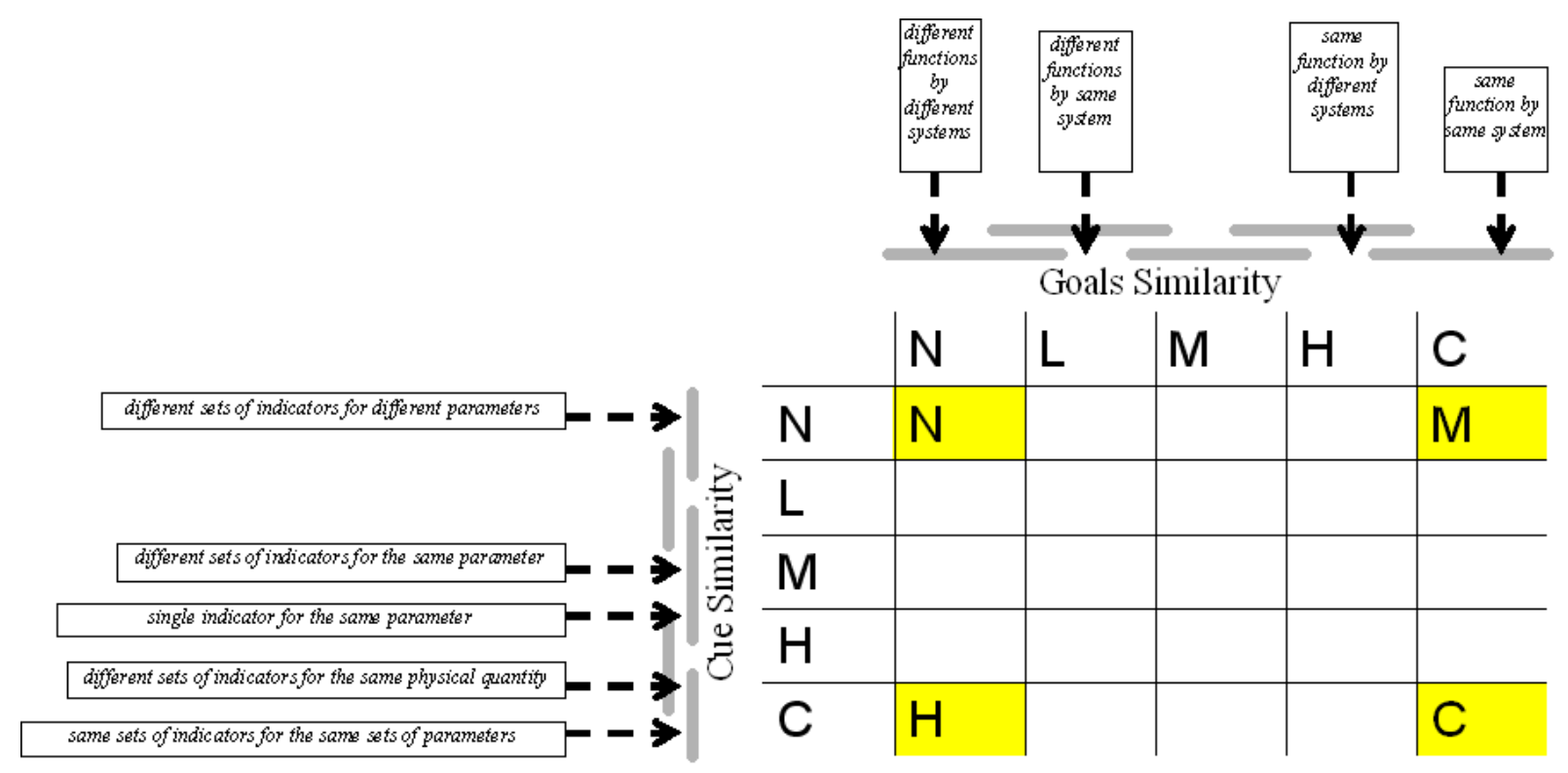

Figure 9. Table of rules for the intermediate variable Task relatedness (partial fill from the expert statements).

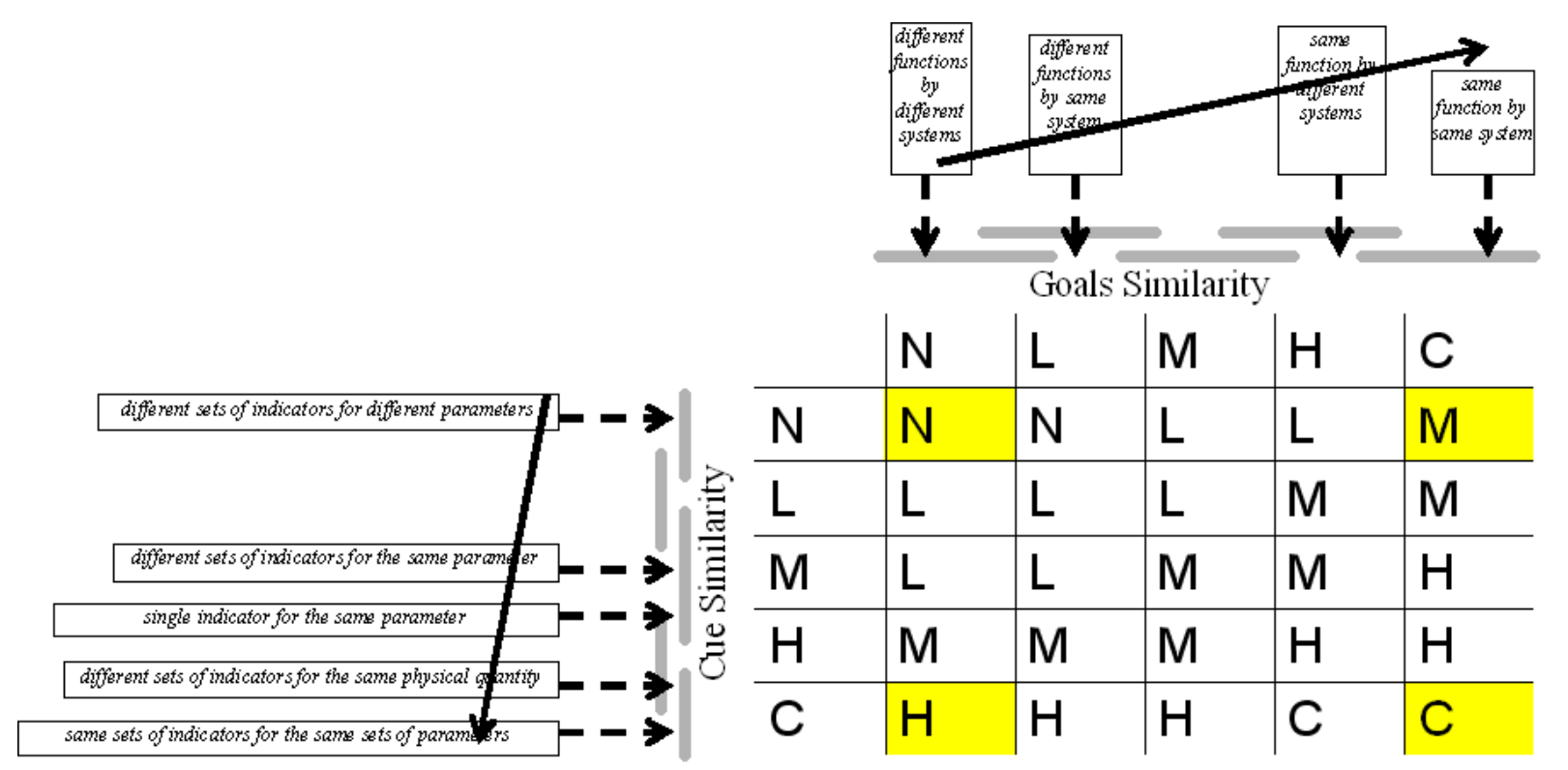

Figure 10. Table of rules for the intermediate variable Task relatedness (complete fill by rules interpolation). 


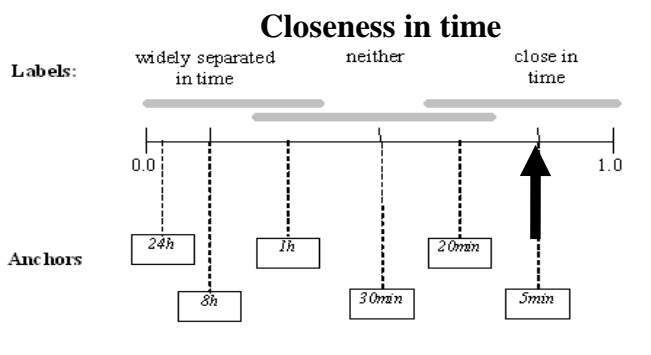

Similarity of functions/goals

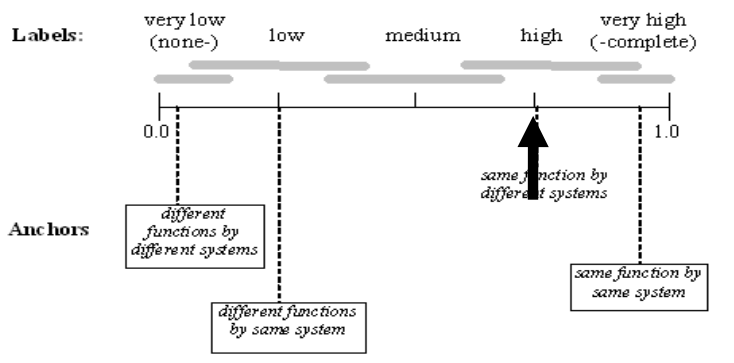

Similarity of cues

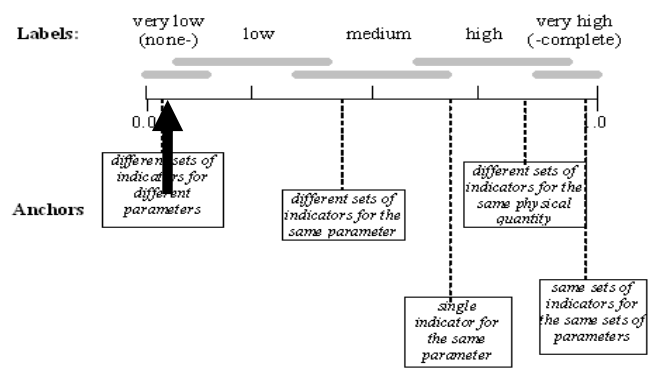

Similarity of performers

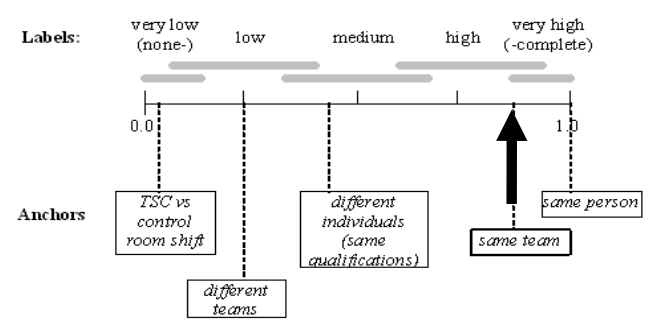

Figure 11. Analyst judgments on input factors for failure to control low pressure injection given failure to inhibit ADS - case 1: judgments given as point values matching the anchors.
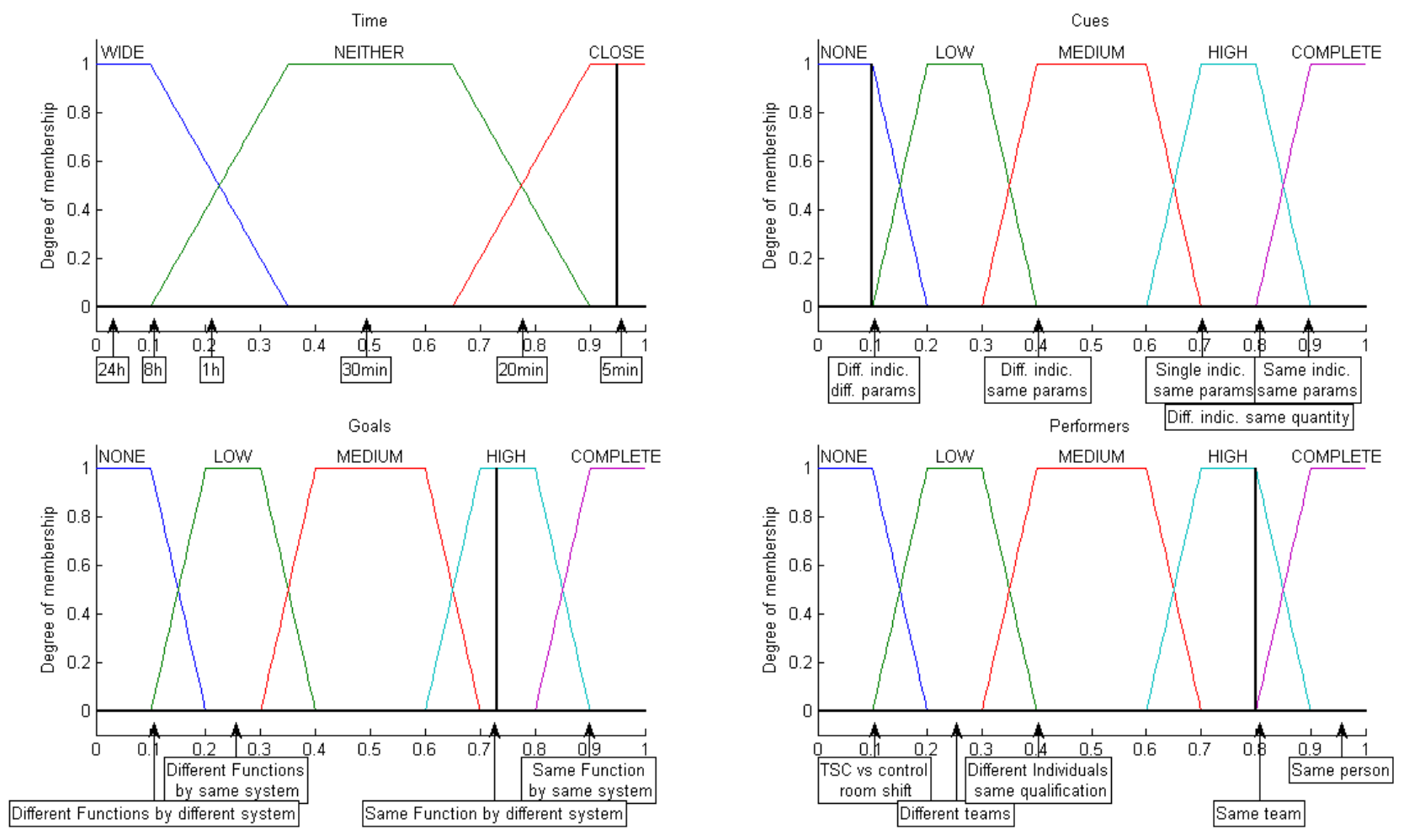

Figure 12. Fuzzy input with trapezoidal MFs for case 1: point estimates matching the anchors. 


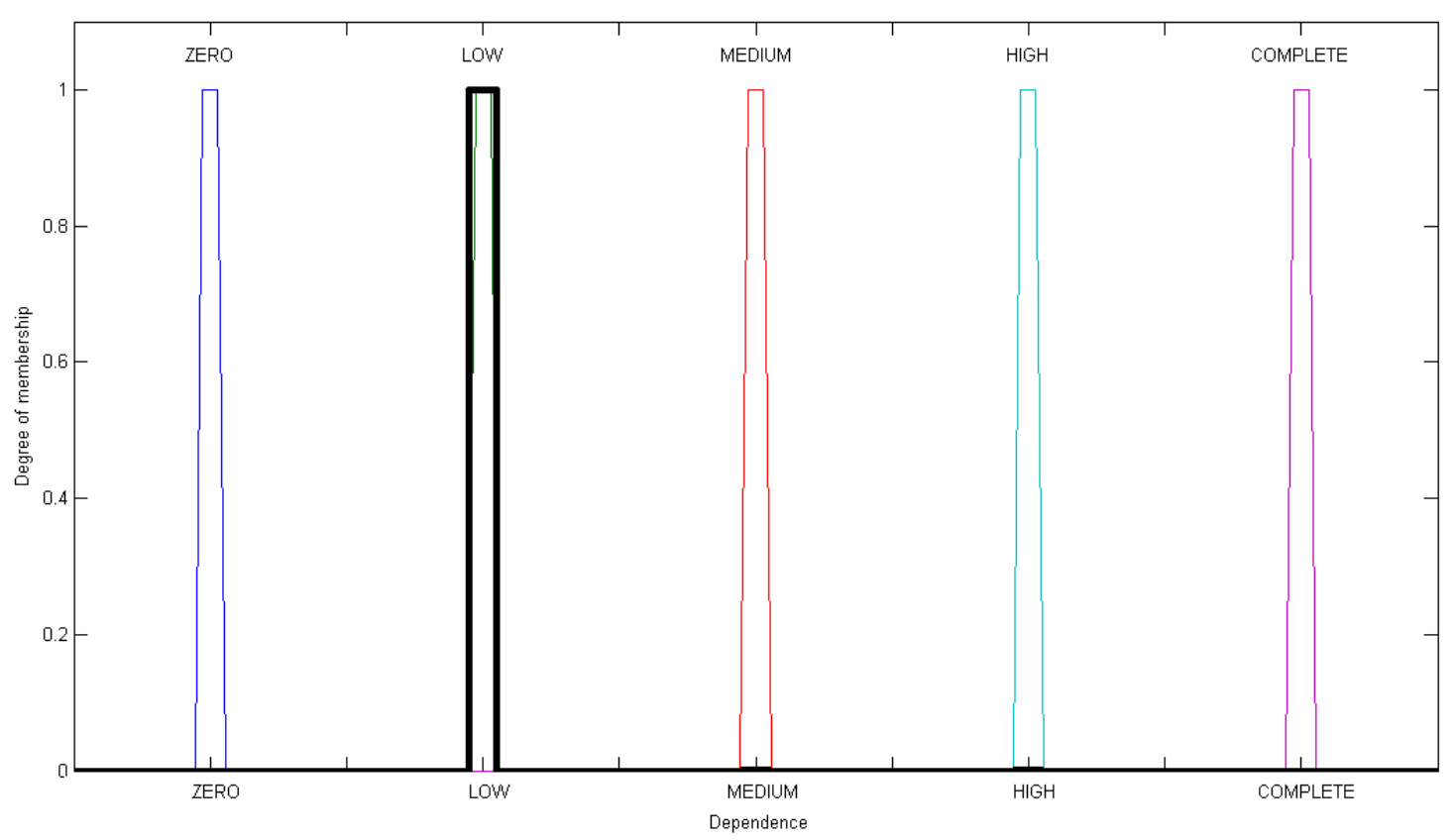

Figure 13. Output of dependence level for case 1: point estimates matching the anchors. 


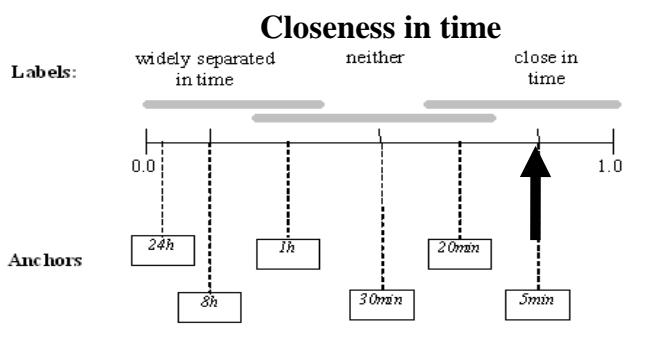

Similarity of functions/goals

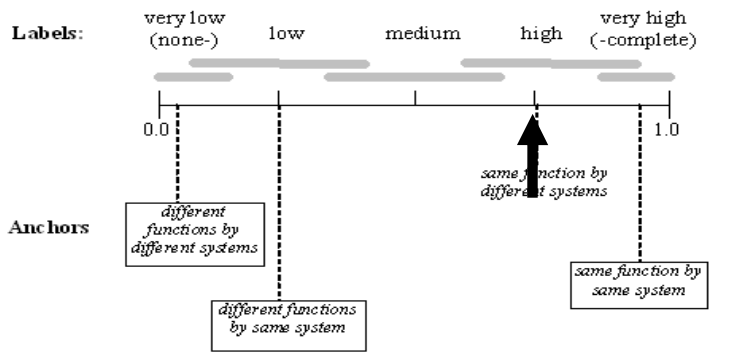

Similarity of cues

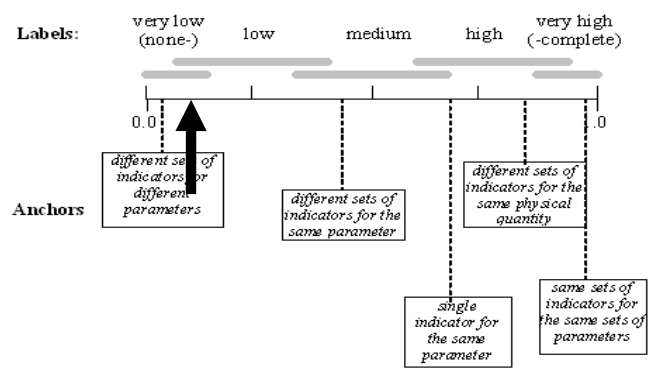

Similarity of performers

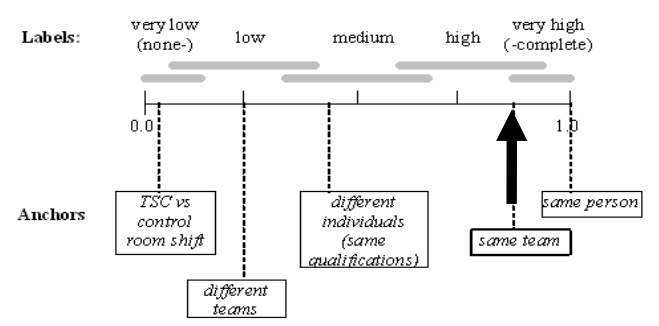

Figure 14. Analyst input on the anchored scale for case 2: point estimates between anchors and labels.
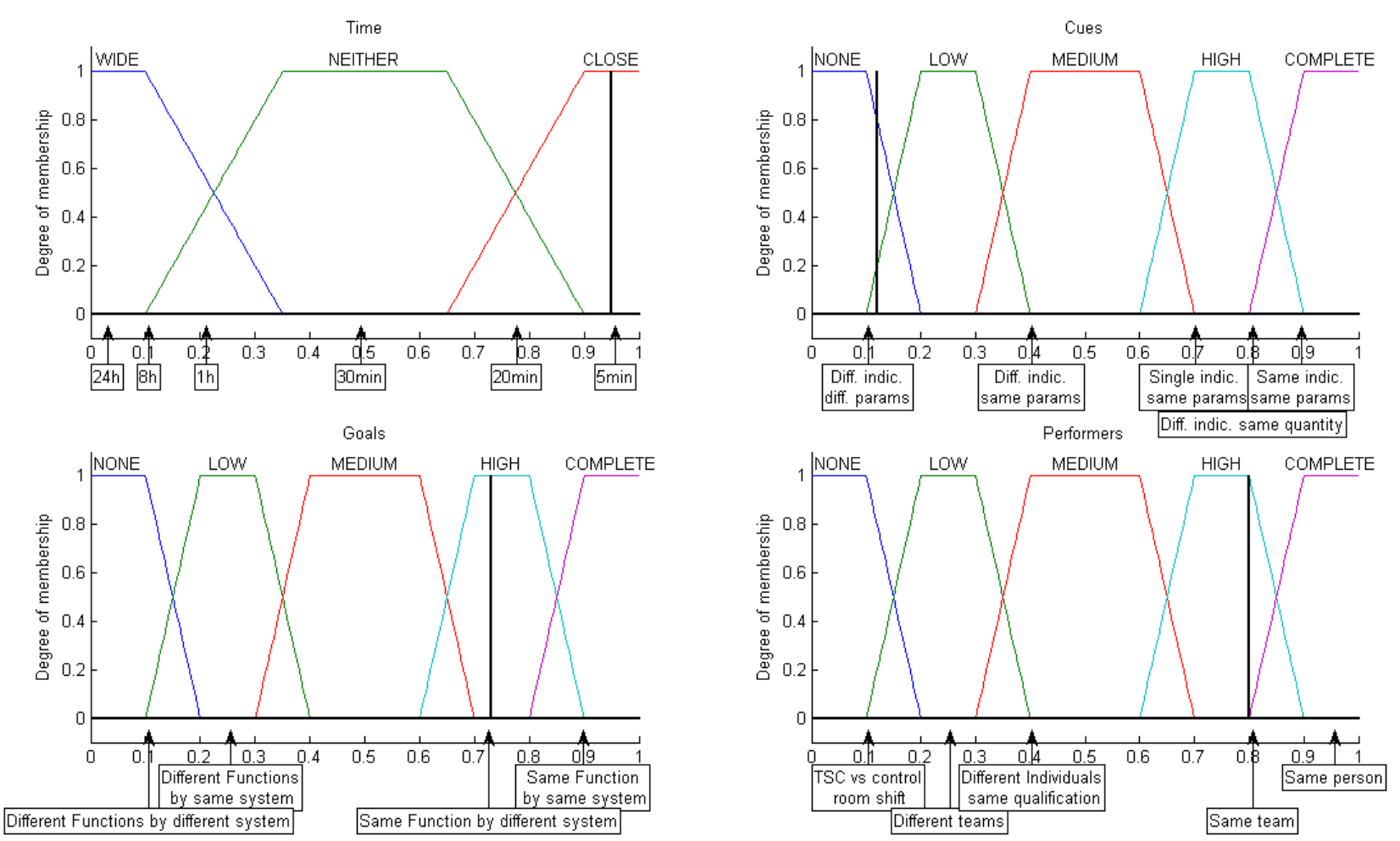

Figure 15. Fuzzy input with trapezoidal MFs for case 2: point estimates between anchors and labels. 


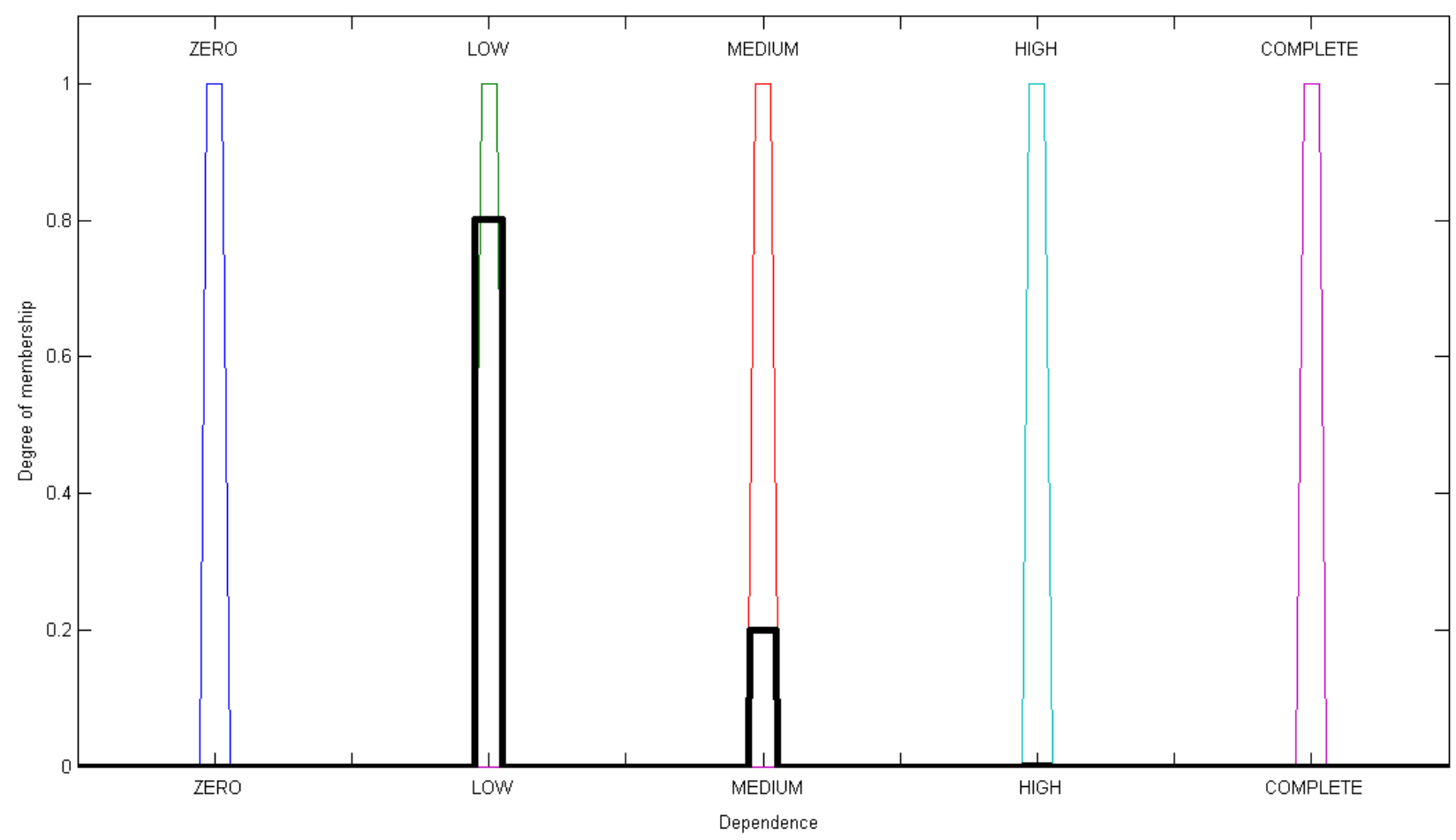

Figure 16. Output of dependence level for case 2: point estimates between anchors and labels. 


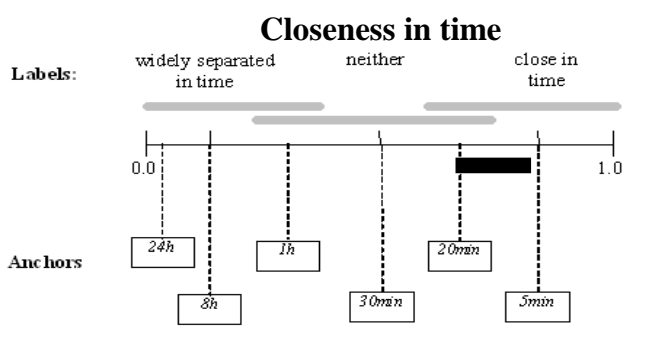

Similarity of functions/goals

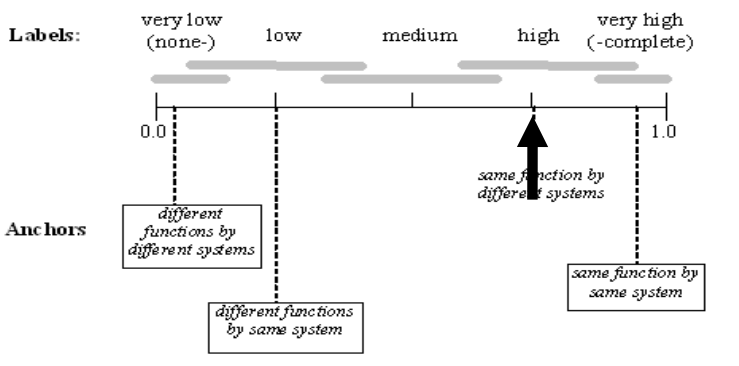

Similarity of cues

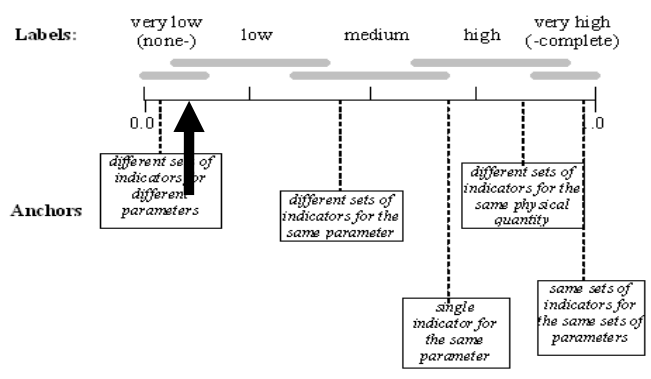

Similarity of performers

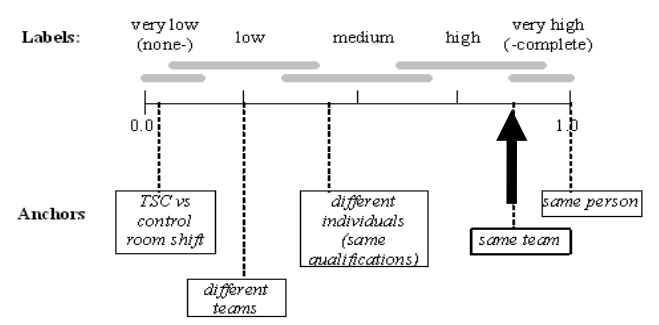

Figure 17. Analyst input on the anchored scale for case 3: range assessment.
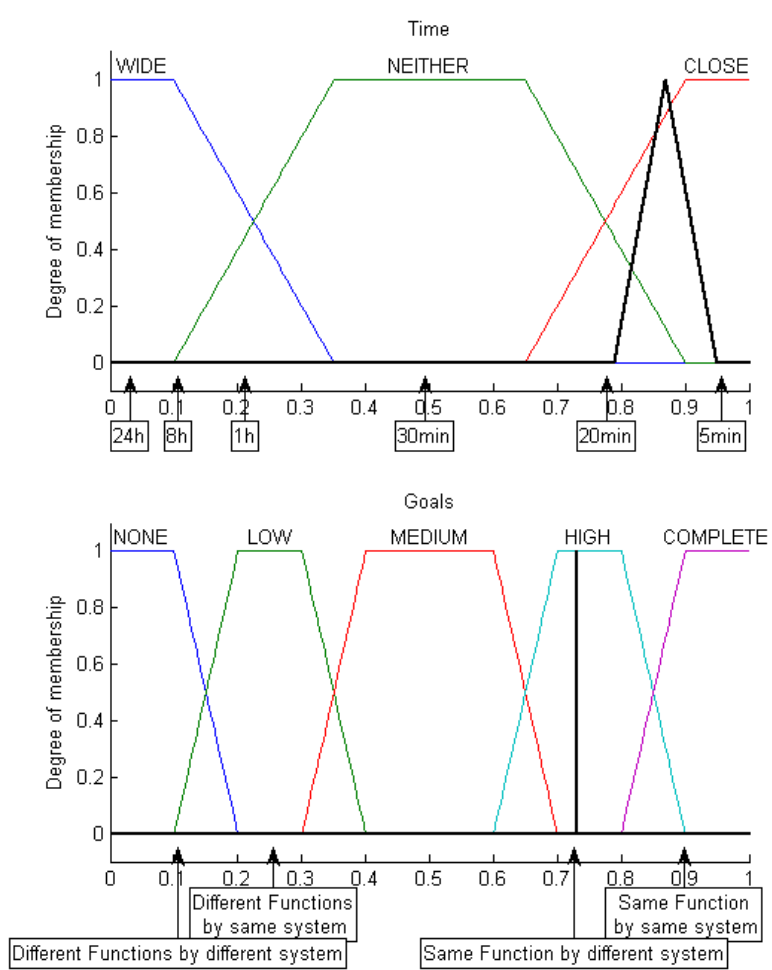

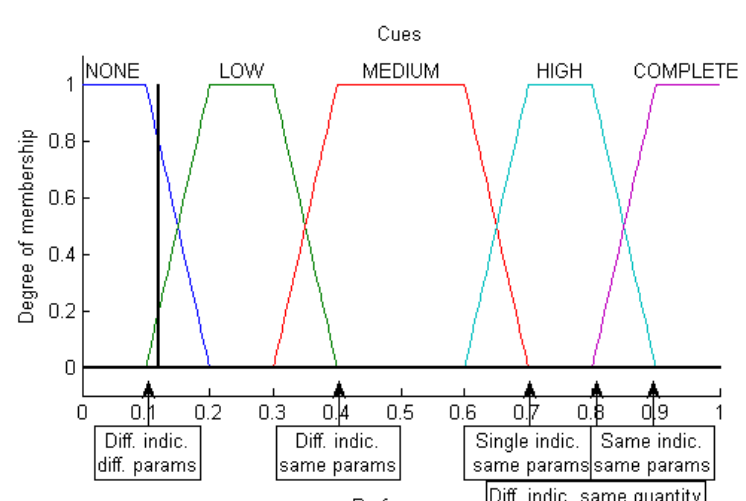

Performers

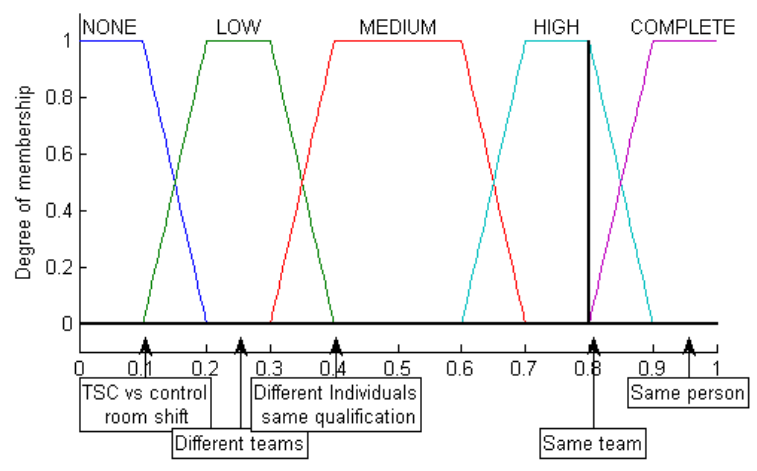

Figure 18. Fuzzy input with trapezoidal MFs for case 3: range assessment. 


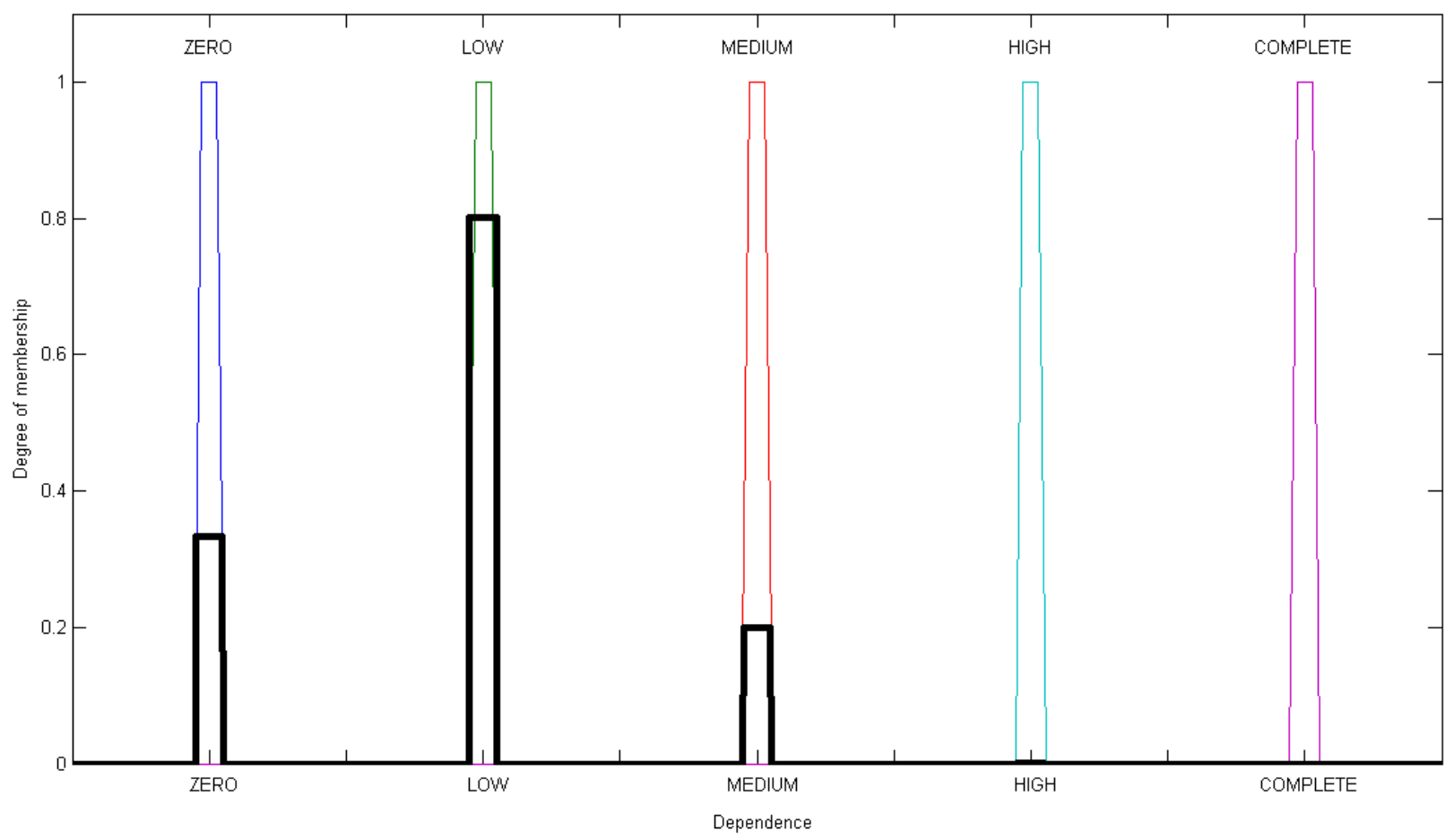

Figure 19. Output of dependence level for case 3: range assessment. 

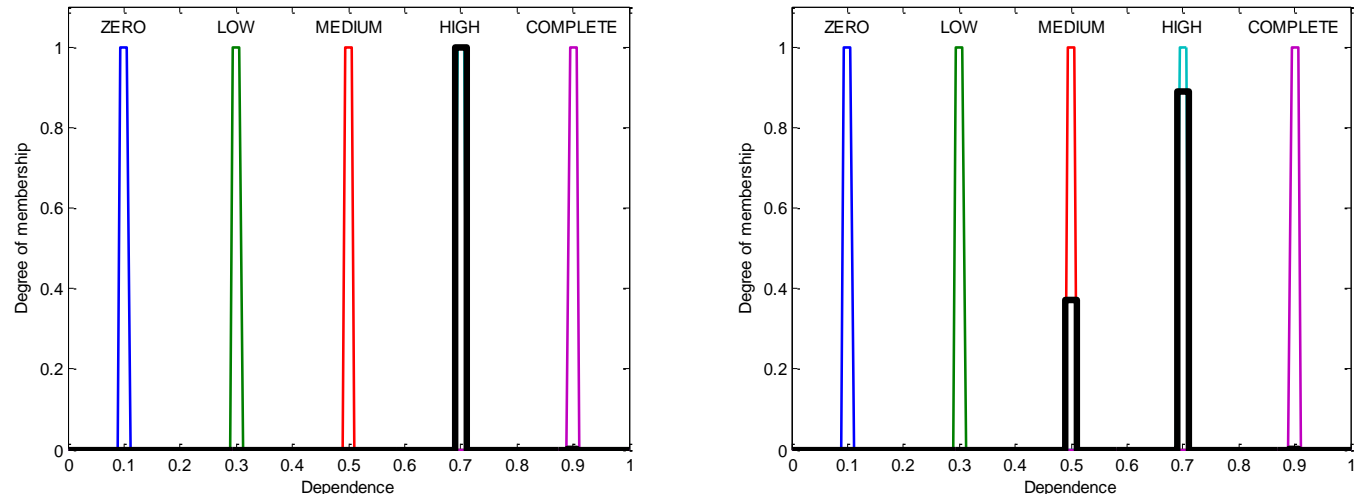

Figure 20. Output of dependence level for cases 4 (left) and 5 (right). 